\title{
STICKY PRICES VERSUS STICKY INFORMATION: DOES IT MATTER FOR POLICY PARADOXES?
}

\author{
Gauti B. Eggertsson \\ Vaishali Garga \\ Working Paper 23961 \\ http://www.nber.org/papers/w23961
NATIONAL BUREAU OF ECONOMIC RESEARCH
1050 Massachusetts Avenue
Cambridge, MA 02138
October 2017

We thank seminar participants at Brown University and the Federal Reserve Bank of Boston for helpful comments and suggestions. All errors are our own.The views expressed herein are those of the authors and do not necessarily reflect the views of the National Bureau of Economic Research.

NBER working papers are circulated for discussion and comment purposes. They have not been peer-reviewed or been subject to the review by the NBER Board of Directors that accompanies official NBER publications.

(C) 2017 by Gauti B. Eggertsson and Vaishali Garga. All rights reserved. Short sections of text, not to exceed two paragraphs, may be quoted without explicit permission provided that full credit, including $\odot$ notice, is given to the source. 
Sticky Prices Versus Sticky Information: Does it Matter for Policy Paradoxes?

Gauti B. Eggertsson and Vaishali Garga

NBER Working Paper No. 23961

October 2017

JEL No. E31,E4,E5,E50,E51,E52,E6,E62

\section{ABSTRACT}

This paper shows that government spending multiplier at the zero lower bound (ZLB) is larger under sticky information than under sticky prices. Similarly, well known paradoxes, e.g., the paradox of toil and the paradox of flexibility become more severe under sticky information. For the case of sticky information it is important to assume that the fiscal policy intervention coincides with the duration of zero interest rates, while such distinction is less important in some special cases for sticky prices. This allows us to unify and clarify results that may appear to contradict each other in the literature.

Gauti B. Eggertsson

Department of Economics

Brown University

64 Waterman Street

Providence, RI 02912

and NBER

gauti_eggertsson@brown.edu

Vaishali Garga

Department of Economics

Brown University

64 Waterman Street

Providence, RI 02912

Vaishali_Garga@brown.edu 


\section{Introduction}

A number of papers documented that fiscal policy is extremely effective to increase demand at the ZLB. In particular the classic government spending multiplier is greater than one, while under normal circumstances it is not, for then monetary policy can do the job via interest rate cuts. Examples include Eggertsson (2011), Christiano, Eichenbaum and Rebelo (2009), Woodford (2011). The literature also uncovered peculiar paradoxes, such as the paradox of toil (Eggertsson (2010)) and the paradox of flexibility (see Bhattarai, Eggertsson and Schoenle (2014) for an overview of this literature and some general results). These results could be interpreted either as a serious challenge to the conventional wisdom or reflect some fundamental flaws of the New Keynesian framework. These results were, however, derived under the assumption that prices are sticky, as in Calvo (1983).

It has long been recognized that the Calvo model of price setting has many peculiar features. This led researchers to explore alternatives, such as information frictions. One of the most prominent proposal to replace the New Keynesian Phillips curve based upon Calvo prices is the assumption of sticky information, proposed by Mankiw and Reis (2001). According to their hypothesis, firms adjust their prices slowly because they do not continously update their information set. Mankiw and Reis argued that this alternative assumption helps explain the data better along certain dimensions. A very natural question, in the light of the radical findings documented in the Calvo model at the ZLB, is if these results carry over to a setting where information rigidities are assumed instead of sticky prices. The main conclusion of this paper is that the answer is yes.

In an important and intriguing recent paper, Kiley (2016), documents experiments in which the fiscal policy results of the Calvo model are overturned upon assuming informational frictions as in Mankiw and Reis. The thought experiment Kiley conducts is as follows: Suppose the Central Bank follows an interest rate peg for 100 periods. What happens if the government increases spending for $1,2, \ldots$ upto 25 periods? What happens if taxes are increased for 1 to 25 periods? Kiley documents that while under Calvo prices the predictions are in line with the existing literature at the ZLB, the predictions are different under informational frictions. In particular, the government spending multiplier is small, tax multiplier is negative and the paradox of flexibility disappears. Kiley's experiment is referred to in this paper as an interest rate peg experiment (PEG-EX). Kiley interprets these findings as suggesting that the sticky-information model is free from policy paradoxes, and thus to be favored over the Calvo model. Here, instead, we argue that these findings are an artifact of the thought experiment considered. The paradoxes in the sticky information framework in fact get even stronger in policy experiments that correspond more closely to those considered in the existing literature.

This paper compares sticky prices and sticky information doing a different experiment. This experiment is identical to the one conducted in Eggertsson (2011), 
Christiano, Eichenbaum and Rebelo (2009), Eggertsson (2010), Woodford (2011). The ZLB is binding due to exogenous fundamental shocks. Once the shocks are over, the policy is given by a simple inflation target (which is missed for the duration of the shocks, due to the ZLB). This experiment is referred to as ZLB experiment (ZLB-EX). This paper documents that the results derived in the literature under sticky prices in the ZLB-EX are even more extreme if sticky prices are replaced with sticky information, which is the opposite of Kiley's result. The government spending multiplier becomes larger, and the paradox of toil and flexibility become more pronounced.

While this may seem to contradict Kiley's findings, it does not. Instead it clarifies that Kiley's PEG-EX is a fundamentally different experiment than done in the existing literature. What is particularly subtle - and interesting - about the comparison, and likely to trigger confusion, is that under sticky prices the ZLB-EX and PEG-EX lead to exactly the same result. It is only when assuming sticky information that the results of the ZLB-EX and the PEG-EX are different. This does not have anything to do with the nature of the nominal frictions. Instead, it is a consequence of the fact that the sticky-information model has infinite number of endogenous state variables. Meanwhile the Calvo model is purely forward looking. The presence of endogenous state variables in the sticky-information model implies that comparing the reaction of an economy assuming an exogenous interest rate peg, versus the reaction of the economy if the central bank's policy is bounded by zero due to fundamental shocks, leads to very different results. The same does not apply for perfectly forward looking systems like the Calvo model of price stickiness.

This paper first shows analytical examples that clarify the intuition behind these findings. It then moves to numerical examples that replicate Kiley's results. These examples confirm that Kiley's results are driven by the difference in experiments being conducted rather than anything fundamental about the assumption of price stickiness.

Arguably, the ZLB-EX is more economically relevant than PEG-EX. It seems of more limited economic interest - at least in the context of the crisis that started in 2008 - to explore the behavior of New Keynesian models if the short-term interest rate is temporarily pegged for no apparent reasons. Instead, the most economically interesting experiment appears to be when the interest rate is pegged due to the fact that the ZLB is binding on account of a fundamental recessionary shock that prevents the central bank from achieving its objective of stabilizing inflation and output. ${ }^{1}$

\footnotetext{
${ }^{1}$ We contemplate another experiment (Coordinated PEG-EX) in which the duration of the peg is coordinated with the duration of fiscal policy (details in Appendix D). The results of this experiment are qualitatively similar to that of the ZLB-EX. These coordinated pegs have been used by other papers analyzing the effects of fiscal policy as well (see Carlstrom, Fuerst and Paustian (2014)). Exogenous interest rate pegs have been used in McKay, Nakamura and Steinsson (2016), Carlstrom, Fuerst and Paustian (2012) to analyze the forward guidance puzzle, but only in the context of forward-looking models. As we note, in perfectly forward-looking sticky-price models, it does not matter whether one assumes a passive monetary policy due to an exogenous peg or binding ZLB resulting from a fundamental shock. However, it is important to distinguish between Kiley's proposed PEG-EX and Coordinated PEG-EX in the context of
} 
The following table summarizes the numerical results for the two models, calibrated to produce a $10 \%$ drop in output and $2 \%$ annual deflation on impact. It is clear that in the ZLB-EX, government spending is more expansionary at zero interest rate than at positive interest rate and that tax cuts are contractionary at the ZLB, in both the models of nominal stickiness. Moreover, these paradoxes are starker under sticky information.

Table 1: Fiscal multipliers in the sticky-price model

\begin{tabular}{lll}
\hline & Government spending & Tax cut \\
\hline Positive interest rate & 0.42 & 0.36 \\
Zero interest rate & 1.63 & -0.4 \\
\hline
\end{tabular}

Table 2: Fiscal multipliers in the sticky-information model

\begin{tabular}{lll}
\hline & Government spending & Tax cut \\
\hline Positive interest rate & 0.42 & 0.36 \\
Zero interest rate & 4.77 & -2.41 \\
\hline
\end{tabular}

\section{Alternative models of nominal rigidities}

The most commonly used model of nominal rigidities is based on stickiness of prices (see for example,Woodford (2003)). An alternative model of nominal rigidities is based on stickiness of information (Mankiw and Reis (2001)). In the former model, only a fraction of the firms get to reset their prices every period, while in the latter model, all firms get to reset their prices every period but only a fraction of them get to update their information sets in any given period. Both models can be described by a system of 3 equations common in the monetary economics literature - IS curve, AS curve and a policy rule.

In the Calvo model, firms account for the possibility of not being able to reset prices in future periods. Hence, their optimal price decision depends on their current marginal cost as well as current expectation of future marginal costs. On the other hand, in the Mankiw and Reis model, each firm gets to reset its price in every period, but firms that do not get the information update base their price decision on past expectations of current marginal cost. This introduces infinite lags into the model as firms set prices based on different vintages of information.

All of the results in this paper depend on the presence of these lagged variables in the sticky-information framework. But there is nothing special about sticky "information" versus sticky "prices". In fact, the SI model could be interpreted as an overlapping Taylor contract model. Similarly, Calvo originally interpreted the Calvo

sticky-information models as we will see. 
probability as an information friction rather than referring to sticky prices. The models are entirely standard, accordingly, we only report them in their log-linear form, but define all composite parameters, in terms of the underlying deep parameters in Appendix C. Let us denote every variable $x$ in deviation from its steady state as $\hat{x}$, then we get the following equilibria in the two models:

\subsection{Sticky-price approximate equilibrium}

A sticky-price approximate equilibrium, which is accurate up to a first order, is a collection of stochastic process for output, inflation and the short-term interest rate, $\left\{\hat{y}_{t}, \hat{\pi}_{t}, \hat{i}_{t}\right\}$, that solve equations (1) and (2) given (i) a path for taxes and government spending, $\left\{\hat{\tau}_{t}, \hat{g}_{t}\right\}$, determined by fiscal policy, (ii) exogenous shocks corresponding to the efficient rate of interest $\left\{\hat{r}_{t}^{e}\right\}$, and (iii) a specification of monetary policy. ${ }^{2}$

$$
\begin{gathered}
\hat{y}_{t}=\mathbb{E}_{t} \hat{y}_{t+1}-\tilde{\sigma}^{-1}\left(\hat{i}_{t}-\mathbb{E}_{t} \hat{\pi}_{t+1}-\hat{r}_{t}^{e}\right)+\left(\hat{g}_{t}-\mathbb{E}_{t} \hat{g}_{t+1}\right) \\
\hat{\pi}_{t}=\beta \mathbb{E}_{t} \hat{\pi}_{t+1}+\kappa_{y} \hat{y}_{t}-\kappa_{g} \hat{g}_{t}+\kappa_{\tau} \hat{\tau}_{t}
\end{gathered}
$$

Here, $\tilde{\sigma}, \kappa_{y}, \kappa_{g}, \kappa_{\tau}>0,0<\beta \leq 1$ and $\kappa_{y} \geq \kappa_{g}$.

\subsection{Sticky-information approximate equilibrium}

A sticky-information approximate equilibrium, which is accurate up to a first order, is a collection of stochastic process for $\left\{\hat{y}_{t}, \hat{\pi}_{t}, \hat{i}_{t}\right\}$ that solve equations (3) and (4) given (i) a path for $\left\{\hat{\tau}_{t}, \hat{g}_{t}\right\}$ determined by fiscal policy, (ii) exogenous shocks $\left\{\hat{r}_{t}^{e}\right\}$, and (iii) specification of monetary policy.

$$
\begin{gathered}
\hat{y}_{t}=\mathbb{E}_{t} \hat{y}_{t+1}-\tilde{\sigma}^{-1}\left(\hat{i}_{t}-\mathbb{E}_{t} \hat{\pi}_{t+1}-\hat{r}_{t}^{e}\right)+\left(\hat{g}_{t}-\mathbb{E}_{t} \hat{g}_{t+1}\right) \\
\hat{\pi}_{t}=\frac{\lambda}{1-\lambda}\left(\tilde{\kappa}_{y} \hat{y}_{t}-\tilde{\kappa}_{g} \hat{g}_{t}+\tilde{\kappa}_{\tau} \hat{\tau}_{t}\right) \\
+\lambda \sum_{j=0}^{\infty}(1-\lambda)^{j} \mathbb{E}_{t-1-j}\left(\hat{\pi}_{t}+\left[\tilde{\kappa}_{y}\left(\hat{y}_{t}-\hat{y}_{t-1}\right)-\tilde{\kappa}_{g}\left(\hat{g}_{t}-\hat{g}_{t-1}\right)+\tilde{\kappa}_{\tau}\left(\hat{\tau}_{t}-\hat{\tau}_{t-1}\right)\right]\right)
\end{gathered}
$$

Here, $\tilde{\sigma}, \tilde{\kappa}_{y}, \tilde{\kappa}_{g}, \tilde{\kappa}_{\tau}>0,0<\beta \leq 1$ and $\tilde{\kappa}_{y} \geq \tilde{\kappa}_{g}$.

\section{Analytical example}

This section compares the predictions of sticky-price and sticky-information models under the two different experiments described in the introduction - ZLB-EX versus PEG-EX. The results are first summarized and then explained further using simple graphs.

\footnotetext{
${ }^{2}$ Implicitly it is assumed here that there are lump-sum taxes that adjust to clear the government budget constraint, so that fiscal policy is "Ricardian", see Eggertsson (2011) for further discussion.
} 


\subsection{ZLB Experiment (ZLB-EX)}

The ZLB experiment makes the following assumptions:

A1.(Shock): $\hat{r}_{1}^{e}<0, \quad \hat{i}_{1}=\frac{-\bar{i}}{1+\bar{i}}, \quad \hat{r}_{t}^{e}=0$ for $t>1$

A2.(Fiscal policy): $\left(\hat{g}_{1}, \hat{\tau}_{1}\right)=\left(\hat{g}_{s}, \hat{\tau}_{s}\right), \quad\left(\hat{g}_{2}, \hat{\tau}_{2}\right)=(0,0)$

A3.(Monetary policy): $\hat{\pi}_{t}=0$ for $t>1$

A4. $\left(\right.$ Perfect foresight $\left.{ }^{3}\right): \mathbb{E}_{t} \hat{y}_{t+1}=\hat{y}_{t+1}, \quad \mathbb{E}_{t} \hat{\pi}_{t+1}=\hat{\pi}_{t+1} \quad \forall t$

Proposition 1. (ZLB-EX) Under the assumptions of ZLB-EX, the sticky price and sticky information models have the following solutions:

Sticky-price model:

$$
\begin{aligned}
& \hat{y}_{1}=\tilde{\sigma}^{-1}\left[\frac{\bar{i}}{1+\bar{i}}+\hat{r}_{1}^{e}\right]+\hat{g}_{1} \\
& \hat{\pi}_{1}=\left(\kappa_{y}-\kappa_{g}\right) \hat{g}_{1}+\kappa_{y} \tilde{\sigma}^{-1}\left[\frac{\bar{i}}{1+\bar{i}}+\hat{r}_{1}^{e}\right]+\kappa_{\tau} \hat{\tau}_{1} \\
& \hat{i}_{1}=\frac{-\bar{i}}{1+\bar{i}} \\
& \hat{y}_{2}=\hat{\pi}_{2}=\hat{i}_{2}=0 \\
& \hat{y}_{t}=\hat{\pi}_{t}=\hat{i}_{t}=0 \quad \forall t>2
\end{aligned}
$$

Sticky-information model:

$$
\begin{aligned}
& \hat{y}_{1}=\gamma_{1} \hat{g}_{1}+\gamma_{2} \hat{\tau}_{1}+(2-\lambda) \tilde{\sigma}^{-1}\left[\frac{\bar{i}}{1+\bar{i}}+\hat{r}_{1}^{e}\right] \\
& \hat{\pi}_{1}=\gamma_{3} \hat{g}_{1}+\gamma_{4} \hat{\tau}_{1}+\left(\frac{\lambda(2-\lambda) \tilde{\kappa}_{y} \tilde{\sigma}^{-1}}{1-\lambda}\right)\left[\frac{\bar{i}}{1+\bar{i}}+\hat{r}_{1}^{e}\right] \\
& \hat{i}_{1}=\frac{-\bar{i}}{1+\bar{i}} \\
& \hat{y}_{2}=\gamma_{5} \hat{g}_{1}+\gamma_{2} \hat{\tau}_{1}+(1-\lambda) \tilde{\sigma}^{-1}\left[\frac{\bar{i}}{1+\bar{i}}+\hat{r}_{1}^{e}\right] \\
& \hat{\pi}_{2}=0 \\
& \hat{i}_{2}=\tilde{\sigma}\left(\hat{y}_{3}-\hat{y}_{2}\right) \\
& \hat{y}_{t}=\hat{y}_{t-1}\left[\frac{\lambda \Sigma_{j=0}^{t-2}(1-\lambda)^{j}}{\frac{\lambda}{1-\lambda}+\lambda \Sigma_{j=0}^{t-2}(1-\lambda)^{j}}\right] \quad \forall t>2 \\
& \hat{\pi}_{t}=0 \quad \forall t>2 \\
& \hat{i}_{t}=\tilde{\sigma}\left(\hat{y}_{t+1}-\hat{y}_{t}\right) \quad \forall t>2
\end{aligned}
$$

\footnotetext{
${ }^{3}$ In the sticky information model, perfect foresight is assumed only for those agents who update their information set in any given period. Also, this assumption is only for illustration purposes, and is relaxed later in the numerical results section.
} 
where, $\gamma_{1}, \gamma_{2}, \gamma_{3}, \gamma_{4}, \gamma_{5}>0$ are defined as follows:

$$
\begin{aligned}
& \gamma_{1}=\left[\frac{(2-\lambda) \tilde{\kappa}_{y}-(1-\lambda) \tilde{\kappa}_{g}}{\tilde{\kappa}_{y}}\right], \gamma_{2}=\left[\frac{(1-\lambda) \tilde{\kappa}_{\tau}}{\tilde{\kappa}_{y}}\right], \gamma_{3}=\left[\frac{\lambda(2-\lambda)}{1-\lambda}\right]\left(\tilde{\kappa}_{y}-\tilde{\kappa}_{g}\right), \gamma_{4}=\left[\frac{\lambda(2-\lambda)}{1-\lambda}\right] \tilde{\kappa}_{\tau}, \\
& \gamma_{5}=(1-\lambda)\left(\frac{\tilde{\kappa}_{y}-\tilde{\kappa}_{g}}{\tilde{\kappa}_{y}}\right) .
\end{aligned}
$$

Proof. The full details of the proof are provided in Appendix A, which essentially just involves solving the models backwards using A1-A4 and keeping track of all terms.

These equations can be used to solve for the fiscal multipliers. As is well known in the New Keynesian literature, policy paradoxes in the Calvo model with a one-period deterministic fundamental shock take a weaker form. Tax cuts have no effect on output and government spending increases output one-for-one. However, as shown in the following results, even in this one-period shock setting, the sticky-information model exhibits the policy paradoxes. This emphasizes that there is nothing fundamental in the Calvo price stickiness assumption that drives the policy paradoxes.

The following two results follow directly from solving the equations in Proposition 1.

Result 1 (Government spending multipliers). In the ZLB-EX, government spending multiplier at the ZLB is larger in the sticky-information model than in the sticky-price model.

$$
\begin{aligned}
& \left(\frac{d \hat{y}_{1}}{d \hat{g}_{1}}\right)^{S I}=\frac{(2-\lambda) \tilde{\kappa}_{y}-(1-\lambda) \tilde{\kappa}_{g}}{\tilde{\kappa}_{y}} \geq 1 \\
& \left(\frac{d \hat{y}_{1}}{d \hat{g}_{1}}\right)^{S P}=1
\end{aligned}
$$

Result 2 (Paradox of toil). In the ZLB-EX, tax cuts at the ZLB are contractionary in the sticky-information model but have no effect in the sticky-price model.

$$
\begin{aligned}
& \left(\frac{d \hat{y}_{1}}{d \hat{\tau}_{1}}\right)^{S I}=\frac{(1-\lambda) \tilde{\kappa}_{\tau}}{\tilde{\kappa}_{y}} \geq 0 \\
& \left(\frac{d \hat{y}_{1}}{d \hat{\tau}_{1}}\right)^{S P}=0
\end{aligned}
$$

The reason that the paradoxes are more extreme in the sticky information model is the persistence in the effects of the shock. Equation (5) shows that under sticky prices, output, inflation and interest rate return to their steady state values immediately after the fundamental shock is over in period 2. However, under sticky information the recession continues even after the shock is over in period 2 (see equation (6)). This persistence is due to the presence of lagged endogenous variables in the model. The fact that the paradoxes are starker under sticky information can be understood further by contemplating $\hat{y}_{1}$ and $\hat{\pi}_{1}$ graphically, which is done in the next section, before we turn to clarifying Kiley's result, which leads to the opposite conclusion. 


\subsubsection{Discussion}

To simplify the analysis, consider the special case where $\lambda=\frac{\kappa}{1+\kappa}$. The system of equations becomes:

$$
\begin{aligned}
& \text { AS: }\left\{\hat{\pi}_{1}=\kappa_{y} \hat{y}_{1}-\kappa_{g} \hat{g}_{1}+\hat{\kappa}_{\tau} \hat{\tau}_{1} \quad,\right. \text { SP, SI } \\
& \text { AD: } \begin{cases}\hat{y}_{1}=\hat{y}_{2}+\tilde{\sigma}^{-1} \hat{\pi}_{2}+\hat{g}_{1}+\tilde{\sigma}^{-1}\left[\frac{\bar{i}}{1+i}+\hat{r}_{1}^{e}\right] & , \text { SP, SI } \\
\hat{\pi}_{2}=0 & , \text { SP, SI } \\
\hat{y}_{2}=\left\{\begin{array}{lll}
0 & , S P & \\
\gamma_{5} \hat{g}_{1}+\gamma_{2} \hat{\tau}_{1}+(1-\lambda) \tilde{\sigma}^{-1}\left[\frac{\bar{i}}{1+\bar{i}}+\hat{r}_{1}^{e}\right] & , \text { SI }
\end{array}\right.\end{cases}
\end{aligned}
$$

The AS curves across the two models are the same. The AD curves, conditional on expectation of future output, are also the same. This means that the only difference between the multipliers in the two models stems from differences in the expectation of future output. While this expectation is zero in the sticky price (SP) model, it depends on lagged variables in the sticky information (SI) model.

In the $\left(\hat{y}_{1}, \hat{\pi}_{1}\right)$ space, the initial equilibrium for both the models is given by the intersection of the aggregate demand (AD) and aggregate supply (AS) curves at point $\mathrm{E}$ as shown in figure 1 . The AD curves are vertical because demand does not depend on current inflation, only expectation of future inflation, i.e. inflation in period 2. Output is completely demand determined and pinned down by the shocks $\hat{r}_{1}^{e}$ and $\hat{g}_{1}$, and expectation of future output $\hat{y}_{2}$. To the extent that a tax shock does not affect $\hat{y}_{2}$, it does not matter for current output. For a given level of output, then, inflation is determined where the AD curve intersects the AS curve. Now, consider the effects of two different policy interventions in this framework: an increase in government spending and a tax cut. The final effect of policy on output depends on: a) the direct effect of the policy on aggregate demand $\left.\left(\hat{y}_{1}\right), b\right)$ the direct effect of the policy on aggregate supply $\left.\left(\hat{\pi}_{1}\right), \mathrm{c}\right)$ the indirect effect of the policy on aggregate demand via its effect on the expectation of future output $\left(\hat{y}_{2}\right)$.

The effect of government spending increase is illustrated in figure (1a). There is a direct effect on the AS curves in both the models. Government spending takes away resources from private consumption, so people want to work more to make up for the lost consumption, shifting out labor supply and reducing real wages. The lower real wages mean that the firms can produce more at any given rate of inflation, shifting the AS curve out to the right. There is also a direct effect on AD in both the models as government spending increases "autonomous" spending in the economy. In the SP model, an increase in government spending does not interact with inflation expectations, and hence, the real interest rate remains unchanged. This causes consumption to remain unchanged, and all of the increase in government spending shows up onefor-one in output expansion. In the SI model, there is an additional indirect effect of government spending on output, because current government spending becomes 
an endogenous state variable in the model, that produces expectations of a higher future output. The net effect is that there is a rightward shift in AD curves in both the models, but the shift is larger for the SI model. The new equilibria are given by points $\mathrm{A}^{\prime}$ and $\mathrm{B}^{\prime}$ for the SP and SI models, respectively. At these points, output is higher than the initial equilibrium for both SI and SP, but the increase is larger under SI.

The effect of tax cut is illustrated in figure (1b). There is a direct effect on the AS curves in both the models. People want to work more, as they get more money in their pocket for every hour worked. This reduces real wages, the benefits of which get passed onto the consumers, producing deflation in the economy. There is no direct effect on the AD curves in either model. In the purely forward-looking Calvo model, expectation of future output is independent of current fiscal policy. In the SI model, however, there is an indirect effect of tax cut on current output, as current tax cut becomes an endogenous state variable, that produces expectations of a lower future output. This in turn lowers output today. The net effect is that there is no change in $\mathrm{AD}$ in the Calvo model, but a leftward shift in AD in the SI model. The new equilibria are given by points $\mathrm{A}^{\prime \prime}$ and $\mathrm{B}^{\prime \prime}$ for the SP and SI models, respectively. At these points, output is lower in the SI model but remains unchanged in the SP model.

Hence we have just seen analytically and graphically why in the ZLB-EX the policy paradoxes are actually stronger under sticky information than sticky prices. We now turn to how one can obtain the opposite result by considering an alternative thought experiment.

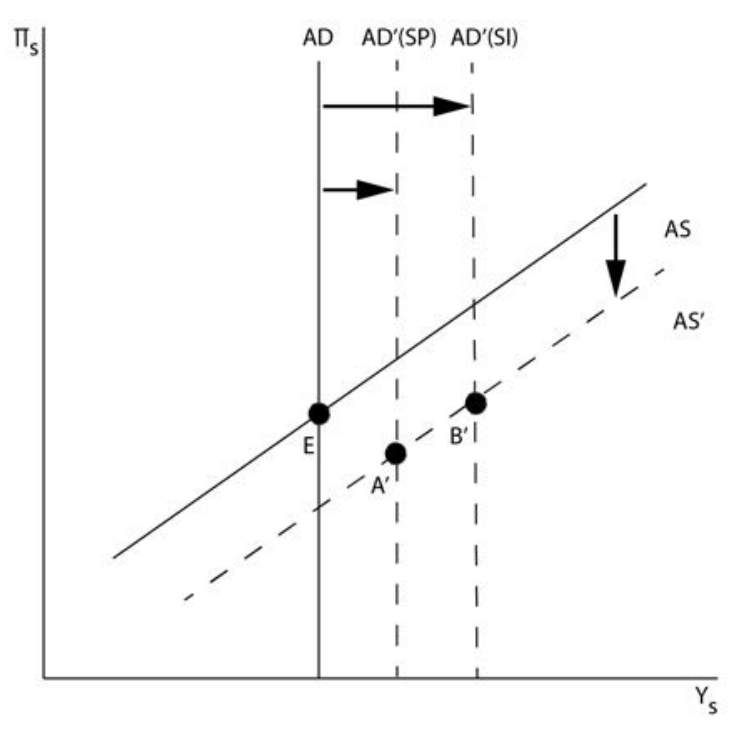

(a) Effect of increasing government spending

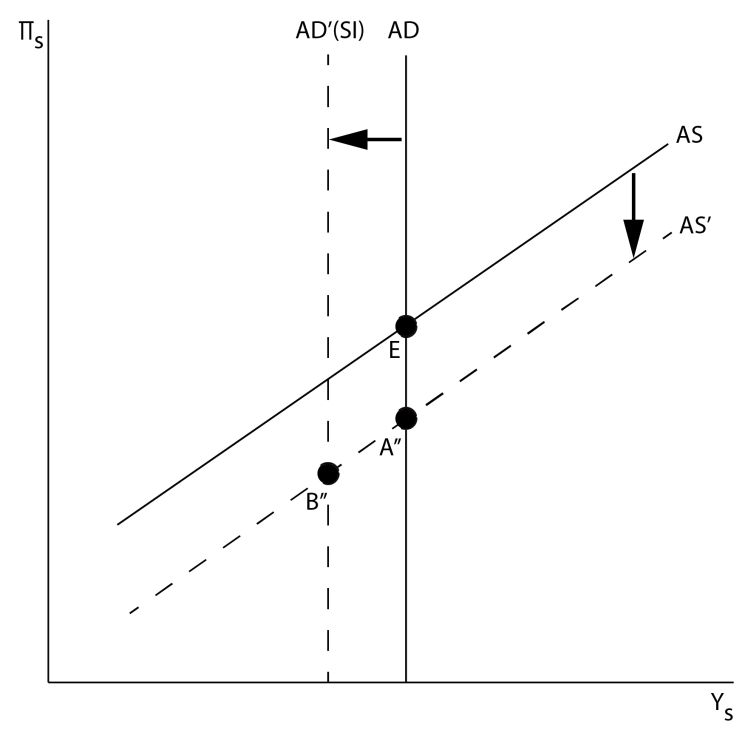

(b) Effect of cutting taxes

Figure 1: Policy predictions of ZLB-EX 


\subsection{Exogenous Interest Rate Peg Experiment (PEG-EX)}

The Exogenous-PEG experiment makes the following assumptions:

B1.(Shock): $\hat{r}_{t}^{e}=0 \quad \forall t, \quad \hat{i}_{1}=\hat{i}_{2}=0$

B2.(Fiscal policy): $\left(\hat{g}_{1}, \hat{\tau}_{1}\right)=\left(\hat{g}_{s}, \hat{\tau}_{s}\right), \quad\left(\hat{g}_{2}, \hat{\tau}_{2}\right)=(0,0)$

B3.(Monetary policy): $\hat{\pi}_{t}=0$ for $t>2$

B4.(Perfect foresight): $\mathbb{E}_{t} \hat{y}_{t+1}=\hat{y}_{t+1}, \quad \mathbb{E}_{t} \hat{\pi}_{t+1}=\hat{\pi}_{t+1} \quad \forall t$

This alternative experiment is different from the ZLB-EX in two main regards. One, there is no fundamental shock to the economy. Two, the peg lasts for two periods while the fiscal policy is active for only one period, which means that in the second period, inflation is not at its target level assuming sticky information while it remains at zero under sticky prices. This difference generates the difference between the two experiments.

Proposition 2. (PEG-EX) Under the assumptions of exogenous PEG-EX, the sticky price and sticky information models have the following solutions:

Sticky-price model:

$$
\begin{aligned}
& \hat{y}_{1}=\hat{g}_{1} \\
& \hat{\pi}_{1}=\left(\kappa_{y}-\kappa_{g}\right) \hat{g}_{1}+\kappa_{\tau} \tau_{1} \\
& \hat{i}_{1}=0 \\
& \hat{y}_{2}=\hat{\pi}_{2}=\hat{i}_{2}=0 \\
& \hat{y}_{t}=\hat{\pi}_{t}=\hat{i}_{t}=0 \quad \forall t>2
\end{aligned}
$$

Sticky-information model:

$$
\begin{aligned}
\hat{y}_{1} & =\zeta_{1} \hat{g}_{s}+\zeta_{2} \hat{\tau}_{s} \\
\hat{\pi}_{1} & =\zeta_{3} \hat{g}_{s}+\zeta_{4} \hat{\tau}_{s} \\
\hat{i}_{1} & =0 \\
\hat{y}_{2} & =0 \\
\hat{\pi}_{2} & =\zeta_{5} \hat{g}_{s}+\zeta_{6} \hat{\tau}_{s} \\
\hat{i}_{2} & =0 \\
\hat{y}_{t} & =\hat{\pi}_{t}=\hat{i}_{t}=0 \quad \forall t>2
\end{aligned}
$$

where, $\zeta_{1}, \zeta_{3}, \zeta_{4}>0$ and $\zeta_{2}, \zeta_{5}, \zeta_{6}<0$ are defined as follows:

$$
\begin{aligned}
& \zeta_{1}=\left(\frac{1+\tilde{\sigma}^{-1}\left(\frac{\lambda}{1-\lambda}\right) \tilde{\kappa}_{g}}{1+\tilde{\sigma}^{-1}\left(\frac{\lambda}{1-\lambda}\right) \tilde{\kappa}_{y}}\right), \zeta_{2}=\left(\frac{-\tilde{\sigma}^{-1}\left(\frac{\lambda}{1-\lambda}\right) \tilde{\kappa}_{\tau}}{1+\tilde{\sigma}^{-1}\left(\frac{\lambda}{1-\lambda}\right) \tilde{\kappa}_{y}}\right), \zeta_{3}=\left(\frac{-\lambda}{1-\lambda}\right)\left[\tilde{\kappa}_{g}-\tilde{\kappa}_{y}\left(\frac{1+\tilde{\sigma}^{-1}\left(\frac{\lambda}{1-\lambda}\right) \tilde{\kappa}_{g}}{1+\tilde{\sigma}^{-1}\left(\frac{\lambda}{1-\lambda}\right) \tilde{\kappa}_{y}}\right)\right], \\
& \zeta_{4}=\left(\frac{-\lambda}{1-\lambda}\right)\left[\tilde{\kappa}_{y}\left(\frac{\tilde{\sigma}^{-1}\left(\frac{\lambda}{1-\lambda}\right) \tilde{\kappa}_{\tau}}{1+\tilde{\sigma}^{-1}\left(\frac{\lambda}{1-\lambda}\right) \tilde{\kappa}_{y}}\right)-\tilde{\kappa}_{\tau}\right], \zeta_{5}=\left(\frac{\lambda}{1-\lambda}\right)\left[\tilde{\kappa}_{g}-\tilde{\kappa}_{y}\left(\frac{1+\tilde{\sigma}^{-1}\left(\frac{\lambda}{1-\lambda}\right) \tilde{\kappa}_{g}}{1+\tilde{\sigma}^{-1}\left(\frac{\lambda}{1-\lambda}\right) \tilde{\kappa}_{y}}\right)\right], \\
& \zeta_{6}=\left(\frac{\lambda}{1-\lambda}\right)\left[\tilde{\kappa}_{y}\left(\frac{\tilde{\sigma}^{-1}\left(\frac{\lambda}{1-\lambda}\right) \tilde{\kappa}_{\tau}}{1+\tilde{\sigma}^{-1}\left(\frac{\lambda}{1-\lambda}\right) \tilde{\kappa}_{y}}\right)-\tilde{\kappa}_{\tau}\right]
\end{aligned}
$$

Proof. The details of the proof are shown in Appendix B. Again, it simply involves 
solving the model backwards, using B1-B4. The key point in the derivation is that we show that under sticky information in PEG-EX, $\hat{y}_{2}=0$ while $\hat{\pi}_{2}$ may be different from zero, which is in contrast with our previous example.

The following two results follow directly from solving the equations in Proposition 2.

Result 3 (Standard government spending multipliers). In the PEG-EX, the government spending multiplier in the sticky-information model is smaller than that in the sticky-price model and is bounded above by 1 .

$$
\begin{aligned}
& \left(\frac{d \hat{y}_{1}}{d \hat{g}_{1}}\right)^{S I}=\frac{1+\tilde{\sigma}^{-1}\left(\frac{\lambda}{1-\lambda}\right) \tilde{\kappa}_{g}}{1+\tilde{\sigma}^{-1}\left(\frac{\lambda}{1-\lambda}\right) \tilde{\kappa}_{y}}<1 \\
& \left(\frac{d \hat{y}_{1}}{d \hat{g}_{1}}\right)^{S P}=1
\end{aligned}
$$

Result 4 (No paradox of toil). In the PEG-EX, tax cuts are expansionary in the stickyinformation model but have no effect in the sticky-price model.

$$
\begin{aligned}
& \left(\frac{d \hat{y}_{1}}{d \hat{\tau}_{1}}\right)^{S I}=\frac{-\tilde{\sigma}^{-1}\left(\frac{\lambda}{1-\lambda}\right) \tilde{\kappa}_{\tau}}{1+\tilde{\sigma}^{-1}\left(\frac{\lambda}{1-\lambda}\right) \tilde{\kappa}_{y}}<0 \\
& \left(\frac{d \hat{y}_{1}}{d \hat{\tau}_{1}}\right)^{S P}=0
\end{aligned}
$$

\subsubsection{Discussion}

To simplify the analysis, consider again the special case where $\lambda=\frac{\kappa}{1+\kappa}$. The system of equations becomes:

$$
\begin{aligned}
& \text { AS: }\left\{\hat{\pi}_{1}=\kappa_{y} \hat{y}_{1}-\kappa_{g} \hat{g}_{1}+\hat{\kappa}_{\tau} \hat{\tau}_{1} \quad,\right. \text { SP, SI } \\
& \text { AD: } \begin{cases}\hat{y}_{1}=\hat{y}_{2}+\tilde{\sigma}^{-1} \hat{\pi}_{2}+\hat{g}_{1} & , \text { SP, SI } \\
\hat{y}_{2}=0 & , \text { SP, SI } \\
\hat{\pi}_{2}= \begin{cases}0 & \\
\zeta_{5} \hat{g}_{s}+\zeta_{6} \hat{\tau}_{s} & , \mathrm{SI}\end{cases} \end{cases}
\end{aligned}
$$

The AS curves across the two models are the same. The AD curves, conditional on expectation of future inflation, are also the same. This means that the only difference between the multipliers in the two models stems from differences in the expectation of future inflation. Contrast this to our previous experiment, where the only difference in $\mathrm{AD}$ was difference in expectation about output in deviation from steady state in period 2 which is in this experiment zero. Meanwhile, the inflation expectation is zero in the sticky-price model, but depends on lagged variables in the sticky-information 
model. The final effect of policy on output depends on: a) the direct effect of the policy on aggregate demand $\left.\left(\hat{y}_{1}\right), b\right)$ the direct effect of the policy on aggregate supply $\left.\left(\hat{\pi}_{1}\right), \mathrm{c}\right)$ the indirect effect of the policy on aggregate demand via its effect on the expectation of future inflation $\left(\hat{\pi}_{2}\right)$.

As shown in figure (2a), an increase in government spending has a direct on the AS curves in that it produces inflation in both the models, as under ZLB-EX. It also has a direct positive effect on AD in both the models as it increases "autonomous" spending in the economy. There is an additional indirect effect of government spending on output in the SI model: government spending today becomes an endogenous state variable in the model - it acts like a cost push shock that produces deflationary expectation in the economy. This tends to reduces output today. The net effect is that there is a rightward shift in AD curves in both the models, but the shift is smaller for the SI model. The new equilibria are given by points $\mathrm{A}^{\prime}$ and $\mathrm{B}^{\prime}$ for the sticky-price and sticky-information models, respectively. At these points, output is higher than the initial equilibrium for both SI and SP, but the increase is larger under SP.

As shown in figure (2b), a tax cut has a direct effect on AS in that it acts like a negative cost push shock and produces deflation in both the models, as under ZLBEX. It has no direct effect on AD in either models. There is an indirect effect on AD in the SI model, which is absent in the Calvo model. This effect in the SI model occurs because a tax cut today becomes an endogenous state variable in the model it acts like a cost push shock that produces inflationary expectations, which inreases output today. The net effect is that there is no change in AD in the Calvo model, but a rightward shift in $\mathrm{AD}$ in the SI model. The new equilibria are given by points $\mathrm{A}^{\prime \prime}$ and $\mathrm{B}^{\prime \prime}$ for the sticky-price and sticky-information models, respectively. At these points, output is higher in the sticky-information model but remains unchanged in the sticky-price model. 


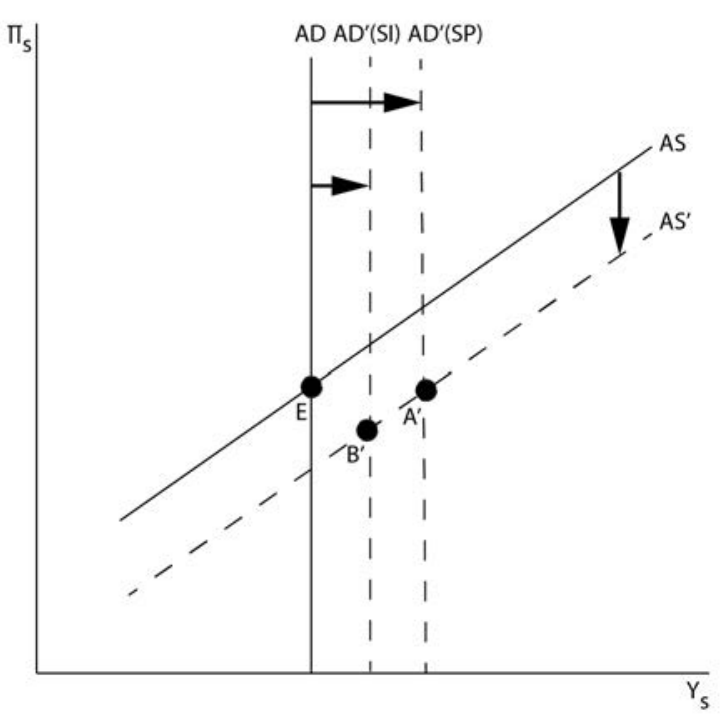

(a) Effect of increasing government spending

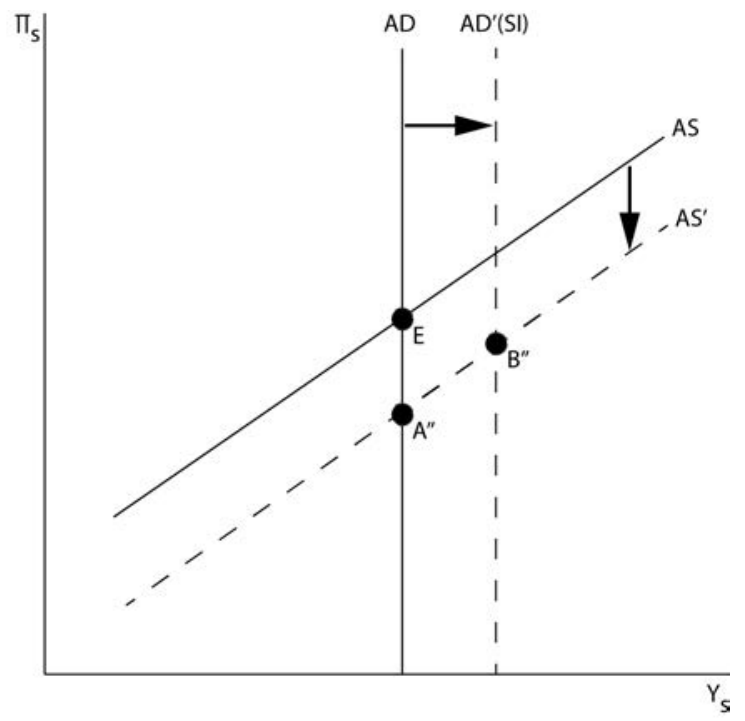

(b) Effect of cutting taxes

Figure 2: Policy predictions of PEG-EX

In summary, in ZLB-EX, the policy paradoxes are present in both sticky-price and sticky-information models, whereas in PEG-EX, the policy paradoxes are absent in the sticky-information model. This difference is a consequence of the fact that the SI model has an infinite number of endogenous state variables while the SP model is purely forward looking. The endogenous state variables become important once one artificially pegs the nominal interest rate independently of the fundamental shocks. This insight will now be carried over to a more dynamic setting, that replicates Kiley's quantitative experiment.

\section{Numerical results: Sticky-price vs sticky-information under ZLB-EX}

This section provides the results of ZLB-EX under a parameter calibration that is common in the literature (details in Appendix C). The aim is to provide a relevant magnitude of the fiscal multipliers and discuss the paradox of flexibility. An AR(1) process is chosen for the natural real interest rate shock and a deterministic transition path is contemplated. The duration of the binding ZLB is endogenously determined. The persistence and magnitude of the real interest rate shock are chosen to produce a $10 \%$ recession and $2 \%$ annual deflation on impact in the sticky-price model. The same shock process is then fed into the the sticky-information model and the information rigidity parameter is chosen to match the $10 \%$ recession and $2 \%$ annual deflation. ${ }^{4}$ This is reminiscent of the exercise in Eggertsson (2011) but the value of the multipliers is different due to a different assumption about the stochastic process - he assumes a

\footnotetext{
${ }^{4}$ Codes for this exercise are available online on our webpage.
} 
two state markov process rather than an AR(1).

\section{Overexpansionary government spending}

Table 3: Government spending multipliers

\begin{tabular}{lll}
\hline & Sticky-price & Sticky-information \\
\hline Positive interest rate & 0.42 & 0.42 \\
Zero interest rate & 1.63 & 4.77 \\
\hline
\end{tabular}

At positive interest rates, government spending crowds out private consumption, and hence its effect on output is small (multiplier less than one) in both sticky price and sticky information models. At zero interest rates, however, government spending is over-expansionary in both the models, with the expansionary effect being higher in the sticky-information model.

\section{Paradox of toil}

Table 4: Tax cut multipliers

\begin{tabular}{lll}
\hline & Sticky-price & Sticky-information \\
\hline Positive interest rate & 0.36 & 0.36 \\
Zero interest rate & -0.4 & -2.41 \\
\hline
\end{tabular}

At positive interest rates, tax cuts promote consumption spending and create an output expansion in both sticky-price and sticky-information models. At zero interest rates, however, tax cuts are contractionary. This contractionary effect is more sharply evident in the sticky-information model.

\section{Paradox of flexibility}

In the numerical solution, as prices become more flexible, the output contraction in response to a negative real interest rate shock becomes worse in both sticky-price and sticky-information models. Infact, the paradox of flexibility is starker under sticky-information than under sticky-prices. This is because increasing information flexibility makes inflation more responsive to the output gap. Therefore, in response to the shock, not only current deflation but also expected deflation is bigger. This expected deflation feeds into the current output and contracts it further. 


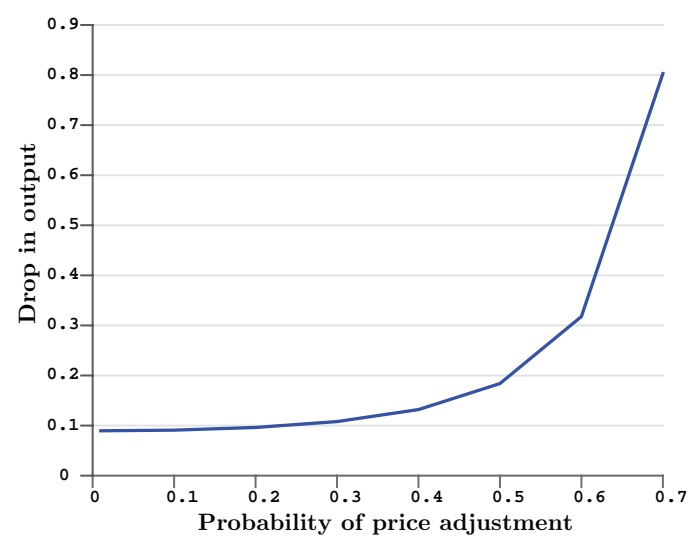

(a) Sticky-price model

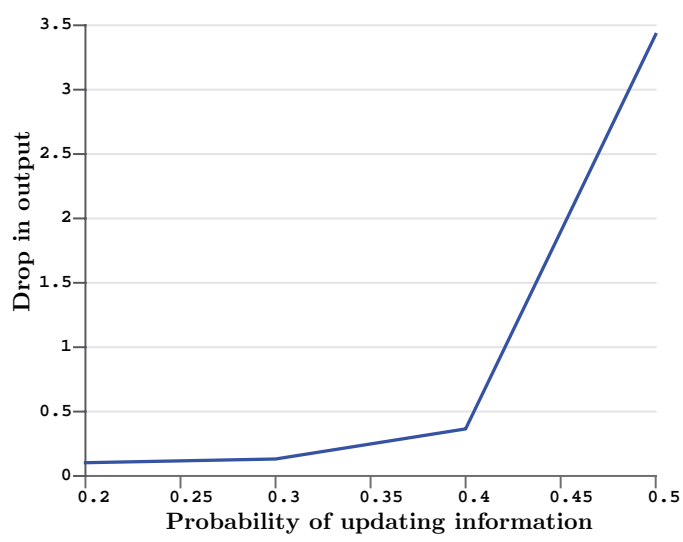

(b) Sticky-information model

Figure 3: Effect of increasing flexibility at zero interest rates

It is well-known what is happening in the sticky-price model, but a brief comment upon this paradox in the sticky-information model is in order, given that it is more subtle due its dependence on the combination of information flexibility $(\lambda)$ and shock persistence $\left(\rho_{r n}\right)$. In a model where the shock persists for the first two periods and goes back to the steady state in the third period, there is a range of $\lambda$ for which the paradox of flexibility holds. This can be shown analytically, but it is rather cumbersome. Similar results can be derived for variations where the shock persists for more than two periods, but it gets progressively harder to get closed form solutions. Accordingly, the finding is presented as a computational result.

Result 5 (Information threshold). Let $\lambda^{*}$ be the threshold level of information-flexibility, beyond which the paradox of flexibility holds. That is, for $\lambda \geq \lambda^{*}, \frac{d y}{d \lambda}<0$. And, let $\rho_{r n}$ be the persistence of the natural interest rate shock. Then, the threshold level of informationflexibility is a decreasing function of the persistence of the shock. That is, $\frac{d \lambda^{*}}{d \rho_{r n}} \leq 0$.

Table 5: Paradox of flexibility and shock persistence

\begin{tabular}{c|c}
\hline$\rho_{r n}$ & $\lambda^{*}$ \\
\hline 0.1 & 1 \\
0.2 & 1 \\
0.3 & 0.76 \\
0.4 & 0.66 \\
0.5 & 0.62 \\
0.6 & 0.48 \\
0.7 & 0.38 \\
0.8 & 0.26 \\
0.9 & 0.18 \\
\hline
\end{tabular}

For any given probability of information updating $\lambda$, paradox of flexibility becomes 
more likely as persistence of the shock increases. This is because, for a given $\lambda$, a negative shock to the natural real interest has a direct effect on current output and inflation - output and inflation fall. There is an additional indirect effect on current output through changes in the expectations of future output. The expectation of future output for every period in which the shock is present will be negative. It is only once the ZLB is no longer binding, that the inflation-targeting central bank will pursue expansionary monetary policy which would increase output. Hence, the final effect on current output depends on the relative magnitude of the cumulative negative output gap in the shock-on periods versus the positive output gap in the shock-off period. The higher the persistence of the shock, the higher is the magnitude of the cumulative negative output gap. This means that as the shock persistence increases, the expansionary effect once the shock is over, is less able to outweigh the contractionary effect while the shock is on. This is true also for the original threshold $\lambda$. Hence, the new threshold $\lambda$ is lower if the persistence of the shock is higher. In the baseline calibration, $\rho_{r n}=0.88$ and the corresponding threshold $\lambda^{*}=0.16$.

\section{Replicating Kiley's results}

Table 6: Calibration parameters (from
\begin{tabular}{cc} 
Parameters & Value \\
\cline { 2 - 3 }$\lambda$ & 0.25 \\
$\beta$ & 1 \\
$\tilde{\sigma}$ & 1 \\
$\kappa_{y}$ & 0.033 \\
$\kappa_{g}$ & 0.0165 \\
$\tilde{\kappa}_{y}$ & 0.1 \\
$\tilde{\kappa}_{g}$ & 0.05 \\
$\phi_{\pi}$ & 1.5 \\
$\phi_{y}$ & 0.125 \\
\hline
\end{tabular}

The PEG-EX is different from the ZLB-EX in two respects. The first is absence of a fundamental shock that drives the economy into the ZLB environment. The second is lack of policy coordination, that is, the duration of the peg is different from the duration of fiscal policy. It is important to understand which of these differences explains the difference in the policy results. Accordingly, we contemplate yet another experiment, which lies somewhere in between the ZLB-EX and the PEG-EX. We call this the Coordinated PEG-EX (CPEG-EX). Under this experiment, there is no fundamental shock to the economy but the duration of policy is coordinated with the duration of the peg.

This section compares the numerical results for multipliers under PEG-EX versus CPEG-EX. Both models are calibrated as per table 6 to replicate the policy results of 


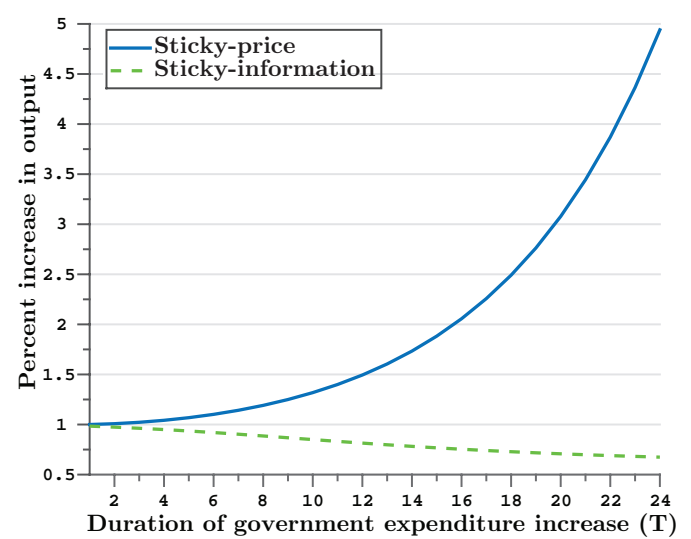

(a) Exogenous PEG-EX

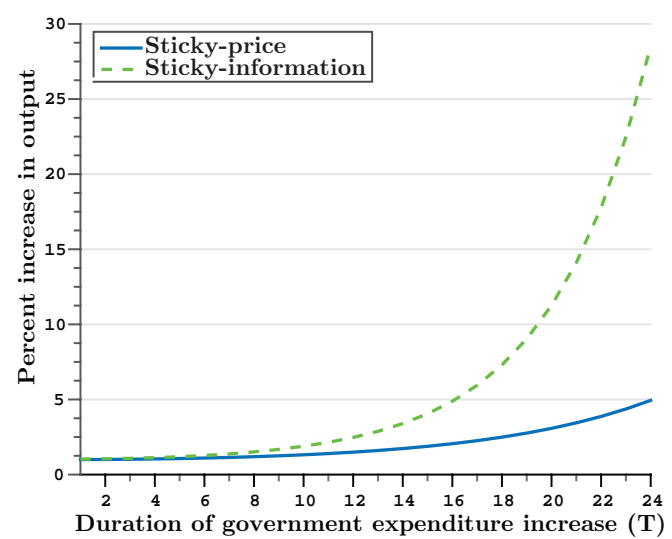

(b) Coordinated PEG-EX

Figure 4: Government spending multiplier

Kiley (2016). The only difference is in the specification of fiscal policy. In particular, under PEG-EX, duration of the fiscal policy intervention is varied period 1 to period 25 , while the interest rate is pegged to its steady state value for 100 periods. Under CPEG-EX, the interest rate peg lasts for as long as the fiscal policy shock is on.

As we will see, the paradox results for the CPEG-EX are qualitatively similar to that of the ZLB-EX. This underlines the importance of policy coordination in this class of models. Quantitatively, the paradoxes are even starker under CPEG-EX than ZLB-EX.

The key result under PEG-EX is that the policy paradoxes depend on the assumptions regarding the nature of price adjustment - sticky prices versus sticky information. Kiley interprets this to mean that changes in assumptions regarding price dynamics can overturn the key policy paradoxes at the ZLB. Figure 4 (a) is a replication of Figure 2 of Kiley's paper. The graph shows that in the sticky-price model, the government expenditure multiplier - that is, the average increase in output over the period of higher government expenditure - is strictly greater than one and increasing in the duration (T) of the fiscal expansion. In the sticky-information model, the government expenditure multiplier is strictly less than one and decreasing in the duration $(\mathrm{T})$ of the fiscal expansion. However, as illustrated in the preceding section, the absence of the over-expansionary effect of government spending under sticky-information here is an artifact of the exogenous interest rate peg. Under CPEG-EX, government spending is over-expansionary even under the assumption of sticky-information, as depicted in Figure 4 (b). Infact, the multiplier is higher under sticky-information than under stick-prices.

Similarly, Figure 5 shows the effect a tax increase under the assumption of sticky-price and sticky-information under PEG-EX versus CPEG-EX. In the sticky-price model, tax increase creates output expansion under both experiments. However, the effect of tax increase on output in the sticky-information model depends on the experiment 
being performed. Under PEG-EX in Figure 5 (a), tax increase has no effect on output, whereas under CPEG-EX in Figure 5 (b), tax increase is expansionary. Infact, the expansionary effect under CPEG-EX is higher in the sticky-information model than in the sticky-price model.

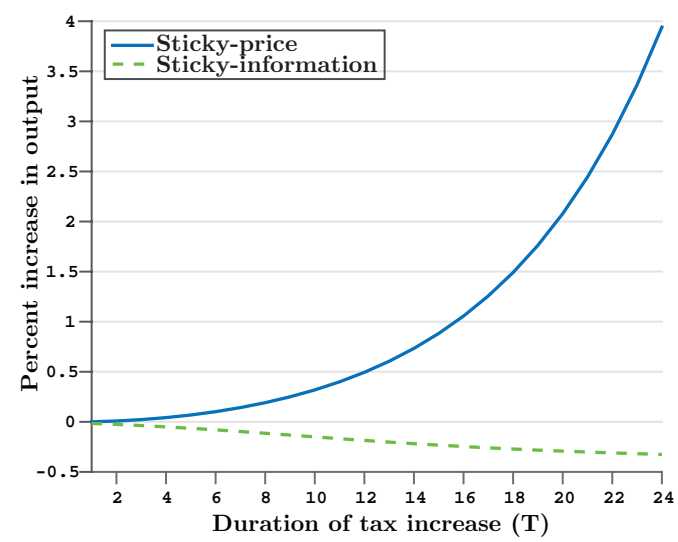

(a) Exogenous PEG-EX

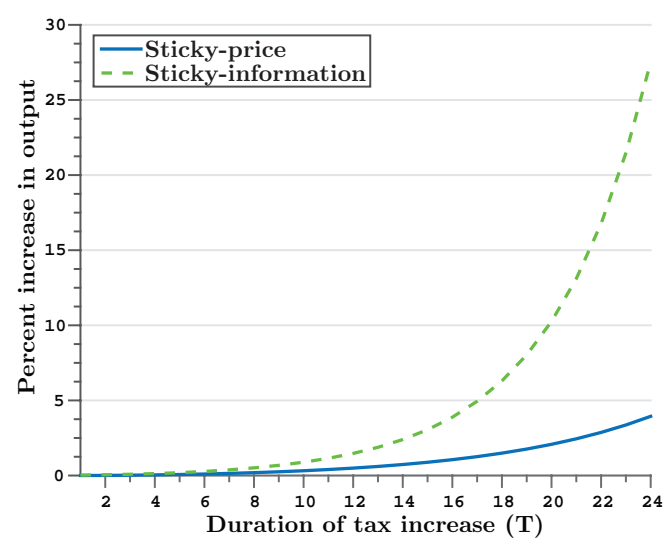

(b) Coordinated PEG-EX

Figure 5: Tax multiplier

These graphs illustrate that keeping the interest rate pegged after the policy intervention is over (PEG-EX) reduces the expansionary power of government spending and makes the paradox of toil disappear in the sticky-information model. This is because, in the absence of the peg, the Central Bank would want to lower the interest rate from second period onwards, in order to stimulate the economy. Constraining the Central Bank's ability to do so through a peg, mechanically engineers a contractionary monetary policy in the model and prevents output from expanding. The subtlety lies in the fact that the interest rate peg produces no such contradiction, relative to ZLB-EX/CPEG-EX, in the sticky-price framework. The sticky-price model is purely forward looking, and as soon as the shock is over, output, inflation and interest rate go back to their respective steady states. Hence, holding the interest rate constant is inconsequential in that model.

\section{Conclusion}

This paper demonstrates that the key policy predictions of the New Keynesian model at the ZLB are independent of the assumption about the nature of price dynamics sticky prices or sticky information. This contradicts the recent findings of Kiley (2016) that the policy paradoxes of the sticky-price model are fragile and disappear under the alternative sticky-information assumption of Mankiw and Reis (2001). Kiley's findings are driven by the nature of the experiment he performs, rather than anything fundamental about sticky information. He assumes an arbitrary interest rate peg independent of any fundamental shock or policy intervention (PEG-EX in the paper). 
This is very different from the policy experiment common in the ZLB literature, where the ZLB is binding due to a fundamental shock and policy is explicitly in response to it (ZLB-EX in the paper). It is even different from much of the literature on interest rate pegs, where it is typically assumed that fiscal intervention lasts as long as the interest rate peg (CPEG-EX).

Analytical examples and numerical simulations are used to illustrate and explain this result. In the ZLB-EX, government spending multiplier is larger and paradox of toil is starker under sticky-information than under sticky-prices. This is because in the purely forward looking sticky-price model, current policy leaves expectations of future variables unchanged. Hence, there is only a "direct" effect of current policy on current output. In the backward looking sticky-information model, however, current policy has an additional "indirect" effect on current output, which comes from its effect on the expectations of future variables.

In the alternative experiment, PEG-EX, government spending multiplier is smaller than one and paradox of toil disappears under sticky-information. This is because an arbitrary interest rate peg is equivalent to engineering a contractionary monetary policy in that framework. In the absence of the peg, an inflation-targeting central bank would lower interest rate in order to stimulate output and inflation. The peg puts a constraint on the central bank's ability to do so. The subtle feature of this experiment is that it doesn't affect the predictions of the sticky-price model. This is because the sticky-price model is purely forward looking, and all variables revert to their steady state once the temporary fiscal policy shocks are over. Hence, pegging the interest rate to its steady state value (even after the shock is over) is inconsequential in that model.

We further contemplate an experiment, which is a hybrid of the ZLB-EX and PEGEX, called CPEG-EX. Under CPEG-EX, while the interest rate peg is arbitrary to the extent that there is no fundamental shock causing it, policy duration is coordinated with the peg duration. The results for the multipliers under CPEG-EX are qualitatively similar to that of the ZLB-EX. This underscores the importance of coordinating fiscal policy with the duration of monetary policy passiveness in this class of experiments.

Finally, this paper illustrates numerically that the paradox of flexibility is also starker under sticky-information. This result is harder to intuit from an analytical example, because the response of output to increased flexibility in the stickyinformation model depends on the persistence of the underlying fundamental rate shock.

Overall, the paradoxical results for fiscal policy multiplier at the ZLB could be interpreted as reflecting a weakness of the Calvo model. This paper, however, clarifies that these paradoxes are not a product of the Calvo assumption, but rather are a fundamental feature of models with nominal rigidities, irrespective of its source, be it sticky prices or sticky information. 


\section{References}

Bhattarai, Saroj, Gauti Eggertsson, and Raphael Schoenle. 2014. "Is increased price flexibility stabilizing? redux." National Bureau of Economic Research.

Calvo, Guillermo A. 1983. "Staggered prices in a utility-maximizing framework." Journal of monetary Economics, 12(3): 383-398.

Carlstrom, Charles T, Timothy S Fuerst, and Matthias Paustian. 2012. "Inflation and output in New Keynesian models with a transient interest rate peg."

Carlstrom, Charles T, Timothy S Fuerst, and Matthias Paustian. 2014. "Fiscal multipliers under an interest rate peg of deterministic versus stochastic duration." Journal of Money, Credit and Banking, 46(6): 1293-1312.

Christiano, Lawrence, Martin Eichenbaum, and Sergio Rebelo. 2009. "When is the government spending multiplier large?" National Bureau of Economic Research.

Eggertsson, Gauti B. 2010. “The paradox of toil." FRB of New York Staff Report, , (433).

Eggertsson, Gauti B. 2011. "What fiscal policy is effective at zero interest rates?" In NBER Macroeconomics Annual 2010, Volume 25. 59-112. University of Chicago Press.

Kiley, Michael T. 2016. "Policy paradoxes in the New Keynesian model." Review of Economic Dynamics, 21: 1-15.

Mankiw, N Gregory, and Ricardo Reis. 2001. "Sticky information versus sticky prices: a proposal to replace the New Keynesian Phillips curve." National Bureau of Economic Research.

McKay, Alisdair, Emi Nakamura, and Jón Steinsson. 2016. “The power of forward guidance revisited." The American Economic Review, 106(10): 3133-3158.

Woodford, Michael. 2003. "Interest rate and prices."

Woodford, Michael. 2011. "Simple analytics of the government expenditure multiplier." American Economic Journal: Macroeconomics, 3(1): 1-35. 


\section{Appendix}

\section{A Proof of Proposition 1}

Sticky-price model

$$
\begin{aligned}
& \hat{y}_{t}=\mathbb{E}_{t} \hat{y}_{t+1}-\tilde{\sigma}^{-1}\left(\hat{i}_{t}-\mathbb{E}_{t} \hat{\pi}_{t+1}-\hat{r}_{t}^{e}\right)+\left(\hat{g}_{t}-\mathbb{E}_{t} \hat{g}_{t+1}\right) \\
& \hat{\pi}_{t}=\beta \mathbb{E}_{t} \hat{\pi}_{t+1}+\kappa_{y} \hat{y}_{t}-\kappa_{g} \hat{g}_{t}+\kappa_{\tau} \hat{\tau}_{t}
\end{aligned}
$$

Assumptions (A1)-(A4) imply that:

For $\mathrm{t}=1$ :

$$
\begin{aligned}
& \hat{y}_{1}=\hat{y}_{2}+\frac{\tilde{\sigma}^{-1} \bar{i}}{1+\bar{i}}+\tilde{\sigma}^{-1} \hat{r}_{1}^{e}+\hat{g}_{1} \\
& \hat{\pi}_{1}=\kappa_{y} \hat{y}_{1}-\kappa_{g} \hat{g}_{1}+\kappa_{\tau} \hat{\tau}_{1}
\end{aligned}
$$

For $\mathrm{t}=2$ :

$$
\begin{aligned}
& \hat{y}_{2}=\hat{y}_{3}-\tilde{\sigma}^{-1} \hat{i}_{2} \\
& \hat{\pi}_{2}=\kappa_{y} \hat{y}_{2}
\end{aligned}
$$

For $\mathrm{t}=3$ :

$$
\begin{aligned}
& \hat{y}_{3}=\hat{y}_{4}-\tilde{\sigma}^{-1} \hat{i}_{3} \\
& \hat{\pi}_{3}=\kappa_{y} \hat{y}_{3}
\end{aligned}
$$

For any $\mathrm{t}>2$ :

$$
\begin{aligned}
& \hat{y}_{t}=\hat{y}_{t+1}-\tilde{\sigma}^{-1} \hat{i}_{t} \\
& \hat{\pi}_{t}=\kappa_{y} \hat{y}_{t}
\end{aligned}
$$

Applying (A3) to period 2 Phillips curve (eqn 13) allows us to solve for $\hat{y}_{2}$ as follows:

$$
\begin{aligned}
\hat{\pi}_{2} & =\kappa_{y} \hat{y}_{2} \\
0 & =\kappa_{y} \hat{y}_{2} \\
\Longrightarrow \hat{y}_{2} & =0
\end{aligned}
$$

Substituting $\hat{y}_{2}$ into period 1 IS curve (eqn 12) gives:

$$
\hat{y}_{1}=\frac{\tilde{\sigma}^{-1} \bar{i}}{1+\bar{i}}+\tilde{\sigma}^{-1} \hat{r}_{t}^{e}+\hat{g}_{1}
$$

Substituting $\hat{y}_{1}$ in period 1 Phillips curve (eqn 12) gives:

$$
\hat{\pi}_{1}=\left(\kappa_{y}-\kappa_{g}\right) \hat{g}_{1}+\frac{\kappa_{y} \tilde{\sigma}^{-1} \bar{i}}{1+\bar{i}}+\kappa_{y} \tilde{\sigma}^{-1} \hat{r}_{1}^{e}+\kappa_{\tau} \hat{\tau}_{1}
$$

Output for every period $t>2$ can be solved from that period's Phillips curve using assumption (A3). We get that for $t>2, \hat{y}_{t}=\hat{\pi}_{t}=0$.

\section{Sticky-information model}

$$
\begin{aligned}
\hat{y}_{t} & =\mathbb{E}_{t} \hat{y}_{t+1}-\tilde{\sigma}^{-1}\left(\hat{i}_{t}-\mathbb{E}_{t} \hat{\pi}_{t+1}-\hat{r}_{t}^{e}\right)+\left(\hat{g}_{t}-\mathbb{E}_{t} \hat{g}_{t+1}\right) \\
\hat{\pi}_{t} & =\frac{\lambda}{1-\lambda}\left(\tilde{\kappa}_{y} \hat{y}_{t}-\tilde{\kappa}_{g} \hat{g}_{t}+\tilde{\kappa}_{\tau} \hat{\tau}_{t}\right) \\
& +\lambda \Sigma_{j=0}^{\infty}(1-\lambda)^{j} \mathbb{E}_{t-1-j}\left(\hat{\pi}_{t}+\left[\tilde{\kappa}_{y}\left(\hat{y}_{t}-\hat{y}_{t-1}\right)-\tilde{\kappa}_{g}\left(\hat{g}_{t}-\hat{g}_{t-1}\right)+\tilde{\kappa}_{\tau}\left(\hat{\tau}_{t}-\hat{\tau}_{t-1}\right)\right]\right)
\end{aligned}
$$

Assumptions (A1)-(A4) imply that: 
For $\mathrm{t}=1$

$$
\begin{aligned}
& \hat{y}_{1}=\hat{y}_{2}+\frac{\tilde{\sigma}^{-1} \bar{i}}{1+\bar{i}}+\tilde{\sigma}^{-1} \hat{r}_{1}^{e}+\hat{g}_{1} \\
& \hat{\pi}_{1}=\frac{\lambda}{1-\lambda}\left(\tilde{\kappa}_{y} \hat{y}_{1}-\tilde{\kappa}_{g} \hat{g}_{1}+\tilde{\kappa}_{\tau} \hat{\tau}_{1}\right)
\end{aligned}
$$

For $\mathrm{t}=2$ :

$$
\begin{aligned}
& \hat{y}_{2}=\hat{y}_{3}-\tilde{\sigma}^{-1} \hat{i}_{2} \\
& \hat{\pi}_{2}=\frac{\lambda}{1-\lambda} \tilde{\kappa}_{y} \hat{y}_{2}+\lambda\left(\hat{\pi}_{2}+\left[\tilde{\kappa}_{y}\left(\hat{y}_{2}-\hat{y}_{1}\right)+\tilde{\kappa}_{g} \hat{g}_{1}-\tilde{\kappa}_{\tau} \hat{\tau}_{1}\right]\right)
\end{aligned}
$$

For $\mathrm{t}=3$ :

$$
\begin{aligned}
& \hat{y}_{3}=\hat{y}_{4}-\tilde{\sigma}^{-1} \hat{i}_{3} \\
& \hat{\pi}_{3}=\frac{\lambda}{1-\lambda} \tilde{\kappa}_{y} \hat{y}_{3}+[\lambda+\lambda(1-\lambda)]\left(\hat{\pi}_{3}+\tilde{\kappa}_{y}\left(\hat{y}_{3}-\hat{y}_{2}\right)\right)
\end{aligned}
$$

For $\mathrm{t}=4$ :

$$
\begin{aligned}
& \hat{y}_{4}=\hat{y}_{5}-\tilde{\sigma}^{-1} \hat{i}_{4} \\
& \hat{\pi}_{4}=\frac{\lambda}{1-\lambda} \tilde{\kappa}_{y} \hat{y}_{4}+\left[\lambda+\lambda(1-\lambda)+\lambda(1-\lambda)^{2}\right]\left(\hat{\pi}_{4}+\tilde{\kappa}_{y}\left(\hat{y}_{4}-\hat{y}_{3}\right)\right)
\end{aligned}
$$

For $\mathrm{t}>2$ :

$$
\begin{aligned}
\hat{y}_{t} & =\hat{y}_{t+1}-\tilde{\sigma}^{-1} \hat{i}_{t} \\
\hat{\pi}_{t} & =\frac{\lambda}{1-\lambda} \tilde{\kappa}_{y} \hat{y}_{t} \\
& +\lambda \Sigma_{j=0}^{t-2}(1-\lambda)^{j}\left(\hat{\pi}_{t}+\tilde{\kappa}_{y}\left(\hat{y}_{t}-\hat{y}_{t-1}\right)\right)
\end{aligned}
$$

Period 2 Phillips curve (eqn 21) along with assumption (A3) can be used to solve for $\hat{y}_{2}$ as follows:

$$
\begin{aligned}
\hat{\pi}_{2} & =\frac{\lambda}{1-\lambda} \tilde{\kappa}_{y} \hat{y}_{2}+\lambda\left(\hat{\pi}_{2}+\left[\tilde{\kappa}_{y}\left(\hat{y}_{2}-\hat{y}_{1}\right)+\tilde{\kappa}_{g} \hat{g}_{1}-\tilde{\kappa}_{\tau} \hat{\tau}_{1}\right]\right) \\
0 & =\frac{\lambda}{1-\lambda} \tilde{\kappa}_{y} \hat{y}_{2}+\lambda\left(0+\left[\tilde{\kappa}_{y}\left(\hat{y}_{2}-\hat{y}_{1}\right)+\tilde{\kappa}_{g} \hat{g}_{1}-\tilde{\kappa}_{\tau} \hat{\tau}_{1}\right]\right) \\
\hat{y}_{2} & =\left(\frac{1-\lambda}{2-\lambda}\right)\left[\hat{y}_{1}-\frac{\tilde{\kappa}_{g}}{\tilde{\kappa}_{y}} \hat{g}_{1}+\frac{\tilde{\kappa}_{\tau}}{\tilde{\kappa}_{y}} \hat{\tau}_{1}\right]
\end{aligned}
$$

Substituting $\hat{y}_{2}$ into period 1 IS curve (eqn 20) gives $\hat{y}_{1}$ :

$$
\hat{y}_{1}=\left[\frac{(2-\lambda) \tilde{\kappa}_{y}-(1-\lambda) \tilde{\kappa}_{g}}{\tilde{\kappa}_{y}}\right] \hat{g}_{1}+\left[\frac{(1-\lambda) \tilde{\kappa}_{\tau}}{\tilde{\kappa}_{y}}\right] \hat{\tau}_{1}+(2-\lambda) \frac{\tilde{\sigma}^{-1} \bar{i}}{1+\bar{i}}+\tilde{\sigma}^{-1}(2-\lambda) \hat{r}_{1}^{e}(26)
$$

Substituting $\hat{y}_{1}$ into period 1 Phillips curve (eqn 20) gives $\hat{\pi}_{1}$ :

$$
\hat{\pi}_{1}=\left(\frac{\lambda(2-\lambda)}{1-\lambda}\right)\left[\left(\tilde{\kappa}_{y}-\tilde{\kappa}_{g}\right) \hat{g}_{1}+\tilde{\kappa}_{\tau} \hat{\tau}_{1}+\tilde{\kappa}_{y} \tilde{\sigma}^{-1} \hat{r}_{1}^{e}+\frac{\tilde{\kappa}_{y} \tilde{\sigma}^{-1} \bar{i}}{1+\bar{i}}\right]
$$

To write $\hat{y}_{2}$ in terms of exogenous parameters, substitute the solution for $\hat{y}_{1}$ (eqn 26) into $\hat{y}_{2}$ (eqn 25):

$$
\hat{y}_{2}=(1-\lambda)\left(\frac{\tilde{\kappa}_{y}-\tilde{\kappa}_{g}}{\tilde{\kappa}_{y}}\right) \hat{g}_{1}+(1-\lambda)\left(\frac{\tilde{\kappa}_{\tau}}{\tilde{\kappa}_{y}}\right) \hat{\tau}_{1}+(1-\lambda) \frac{\tilde{\sigma}^{-1} \bar{i}}{1+\bar{i}}+(1-\lambda) \tilde{\sigma}^{-1} \hat{r}_{1}^{e}
$$

Further, we can solve for the transition dynamics of output in each period using the Phillips curve for 
that period along with assumption (A3). For $t>2$, we get:

$$
\begin{aligned}
0 & =\frac{\lambda}{1-\lambda}\left(\tilde{\kappa_{y}} \hat{y}_{t}\right)+\lambda \Sigma_{j=0}^{t-2}(1-\lambda)^{j}\left(0+\tilde{\kappa_{y}}\left(\hat{y}_{t}-\hat{y}_{t-1}\right)\right) \\
0 & =\left[\frac{\lambda}{1-\lambda}+\lambda \Sigma_{j=0}^{t-2}(1-\lambda)^{j}\right] \tilde{\kappa_{y}} \hat{y}_{t}-\left[\lambda \Sigma_{j=0}^{t-2}(1-\lambda)^{j}\right] \tilde{\kappa_{y}} \hat{y}_{t-1} \\
\hat{y}_{t} & =\left[\frac{\lambda \Sigma_{j=0}^{t-2}(1-\lambda)^{j}}{\frac{\lambda}{1-\lambda}+\lambda \Sigma_{j=0}^{t-2}(1-\lambda)^{j}}\right] \hat{y}_{t-1}
\end{aligned}
$$

\section{B Proof of Proposition 4}

\section{Sticky-price model}

$$
\begin{aligned}
& \hat{y}_{t}=\mathbb{E}_{t} \hat{y}_{t+1}-\tilde{\sigma}^{-1}\left(\hat{i}_{t}-\mathbb{E}_{t} \hat{\pi}_{t+1}-\hat{r}_{t}^{e}\right)+\left(\hat{g}_{t}-\mathbb{E}_{t} \hat{g}_{t+1}\right) \\
& \hat{\pi}_{t}=\beta \mathbb{E}_{t} \hat{\pi}_{t+1}+\kappa_{y} \hat{y}_{t}-\kappa_{g} \hat{g}_{t}+\kappa_{\tau} \hat{\tau}_{t}
\end{aligned}
$$

Assumptions (B1)-(B4) imply that:

For $\mathbf{t}=1$

$$
\begin{aligned}
& \hat{y}_{1}=\hat{y}_{2}+\tilde{\sigma}^{-1} \hat{\pi}_{2}+\hat{g}_{1} \\
& \hat{\pi}_{1}=\beta \hat{\tau}_{2}+\kappa_{y} \hat{y}_{1}-\kappa_{g} \hat{g}_{1}+\kappa_{\tau} \hat{\tau}_{1}
\end{aligned}
$$

For $\mathrm{t}=2$ :

$$
\begin{aligned}
& \hat{y}_{2}=\hat{y}_{3} \\
& \hat{\pi}_{2}=\kappa_{y} \hat{y}_{2}
\end{aligned}
$$

For $\mathrm{t}=3$ :

$$
\begin{aligned}
& \hat{y}_{3}=\hat{y}_{4}-\tilde{\sigma}^{-1} \hat{i}_{3} \\
& \hat{\pi}_{3}=\kappa_{y} \hat{y}_{3}
\end{aligned}
$$

For $\mathrm{t}>2$ :

$$
\begin{aligned}
& \hat{y}_{t}=\hat{y}_{t+1}-\tilde{\sigma}^{-1} \hat{i}_{t} \\
& \hat{\pi}_{t}=\kappa_{y} \hat{y}_{t}
\end{aligned}
$$

Period 3 Phillips curve along with assumption (B3) allows us to solve for $\hat{y}_{3}$ as follows:

$$
\begin{aligned}
\hat{\pi}_{3} & =\kappa_{y} \hat{y}_{3} \\
0 & =\kappa_{y} \hat{y}_{3} \\
\Longrightarrow \hat{y}_{3} & =0
\end{aligned}
$$

Substituting $\hat{y}_{3}$ into period 2 IS curve (eqn 32) implies:

$$
\hat{y}_{2}=\hat{y}_{3}=0
$$

Substituting $\hat{y}_{2}$ into period 1 IS curve (eqn 31) gives $\hat{y}_{1}$ :

$$
\hat{y}_{1}=\hat{g}_{1}
$$

Finally, substituting $\hat{y}_{1}$ into period 1 Phillips curve (eqn 31$)$ gives:

$$
\hat{\pi}_{1}=\left(\kappa_{y}-\kappa_{g}\right) \hat{g}_{1}+\kappa_{\tau} \tau_{1}
$$

Further, we can solve for output in each period $t>2$ using the Phillips curve for that period along with assumption (B3). For $\mathrm{t}>2$, we get $\hat{y}_{t}=0$ as $\hat{\pi}_{t}=0$. 


\section{Sticky-information model}

$$
\begin{aligned}
\hat{y}_{t} & =\mathbb{E}_{t} \hat{y}_{t+1}-\tilde{\sigma}^{-1}\left(\hat{i}_{t}-\mathbb{E}_{t} \hat{\pi}_{t+1}-\hat{r}_{t}^{e}\right)+\left(\hat{g}_{t}-\mathbb{E}_{t} \hat{g}_{t+1}\right) \\
\hat{\pi}_{t} & =\frac{\lambda}{1-\lambda}\left(\tilde{\kappa}_{y} \hat{y}_{t}-\tilde{\kappa}_{g} \hat{g}_{t}+\tilde{\kappa}_{\tau} \hat{\tau}_{t}\right) \\
& +\lambda \Sigma_{j=0}^{\infty}(1-\lambda)^{j} \mathbb{E}_{t-1-j}\left(\hat{\pi}_{t}+\left[\tilde{\kappa}_{y}\left(\hat{y}_{t}-\hat{y}_{t-1}\right)-\tilde{\kappa}_{g}\left(\hat{g}_{t}-\hat{g}_{t-1}\right)+\tilde{\kappa}_{\tau}\left(\hat{\tau}_{t}-\hat{\tau}_{t-1}\right)\right]\right)
\end{aligned}
$$

Assumptions (B1)-(B4) imply that:

For $\mathrm{t}=1$

$$
\begin{aligned}
& \hat{y}_{1}=\hat{y}_{2}+\tilde{\sigma}^{-1} \hat{\pi}_{2}+\hat{g}_{1} \\
& \hat{\pi}_{1}=\frac{\lambda}{1-\lambda}\left(\tilde{\kappa}_{y} \hat{y}_{1}-\tilde{\kappa}_{g} \hat{g}_{1}+\tilde{\kappa}_{\tau} \hat{\tau}_{1}\right)
\end{aligned}
$$

For $\mathrm{t}=2$ :

$$
\begin{aligned}
& \hat{y}_{2}=\hat{y}_{3} \\
& \hat{\kappa}_{2}=\frac{\lambda}{1-\lambda} \tilde{\kappa}_{y} \hat{y}_{2}+\lambda\left(\hat{\pi}_{2}+\left[\tilde{\kappa}_{y}\left(\hat{y}_{2}-\hat{y}_{1}\right)+\tilde{\kappa}_{g} \hat{g}_{1}-\tilde{\kappa}_{\tau} \hat{\tau}_{1}\right]\right)
\end{aligned}
$$

For $\mathrm{t}=3$ :

$$
\begin{aligned}
& \hat{y}_{3}=\hat{y}_{4}-\tilde{\sigma}^{-1} \hat{i}_{3} \\
& \hat{\pi}_{3}=\frac{\lambda}{1-\lambda} \tilde{\kappa}_{y} \hat{y}_{3}+[\lambda+\lambda(1-\lambda)]\left(\hat{\pi}_{2}+\tilde{\kappa}_{y}\left(\hat{y}_{3}-\hat{y}_{2}\right)\right)
\end{aligned}
$$

For $\mathrm{t}=4$ :

$$
\begin{aligned}
& \hat{y}_{4}=\hat{y}_{5}-\tilde{\sigma}^{-1} \hat{i}_{4} \\
& \hat{\pi}_{4}=\frac{\lambda}{1-\lambda} \tilde{\kappa}_{y} \hat{y}_{4}+\left[\lambda+\lambda(1-\lambda)+\lambda(1-\lambda)^{2}\right]\left(\hat{\pi}_{4}+\tilde{\kappa}_{y}\left(\hat{y}_{4}-\hat{y}_{3}\right)\right)
\end{aligned}
$$

For $\mathrm{t}>2$ :

$$
\begin{aligned}
\hat{y}_{t} & =\hat{y}_{t+1}-\tilde{\sigma}^{-1} \hat{i}_{t} \\
\hat{\pi}_{t} & =\frac{\lambda}{1-\lambda} \tilde{\kappa}_{y} \hat{y}_{t} \\
& +\lambda \Sigma_{j=0}^{t-2}(1-\lambda)^{j}\left(\hat{\pi}_{t}+\tilde{\kappa}_{y}\left(\hat{y}_{t}-\hat{y}_{t-1}\right)\right)
\end{aligned}
$$

Period 3 Phillips curve (eqn 42) along with assumption (B3) allows us to solve for $\hat{y}_{3}$ as follows:

$$
\begin{aligned}
\hat{\pi}_{3} & =\frac{\lambda}{1-\lambda} \tilde{\kappa}_{y} \hat{y}_{3}+\lambda\left(\hat{\pi}_{3}+\tilde{\kappa}_{y}\left(\hat{y}_{2}-\hat{y}_{2}\right)\right)+\lambda(1-\lambda)\left(\hat{\pi}_{3}+\tilde{\kappa}_{y}\left(\hat{y}_{3}-\hat{y}_{2}\right)\right) \\
0 & =\frac{\lambda}{1-\lambda} \tilde{\kappa}_{y} \hat{y}_{3}+\lambda \mathbb{E}_{t-1}\left(0+\tilde{\kappa}_{y}\left(\hat{y}_{2}-\hat{y}_{2}\right)\right)+\lambda(1-\lambda)\left(0+\tilde{\kappa}_{y}\left(\hat{y}_{3}-\hat{y}_{2}\right)\right) \\
\hat{y}_{3} & =\hat{y}_{2}\left[\frac{\lambda+\lambda(1-\lambda)}{\frac{\lambda}{1-\lambda}+\lambda+\lambda(1-\lambda)}\right]
\end{aligned}
$$

Substituting $\hat{y}_{3}$ into period 2 IS curve (eqn 41) gives the following equation for $\hat{y}_{2}$ :

$$
\hat{y}_{2}=\hat{y}_{2}\left[\frac{\lambda+\lambda(1-\lambda)}{\frac{\lambda}{1-\lambda}+\lambda+\lambda(1-\lambda)}\right]
$$

Since $\lambda>0$, the only solution to this equation is $\hat{y}_{2}=0$. 
Substituting $\hat{y}_{2}=0$ into period 1 IS curve and period 2 Phillips curve gives:

$$
\begin{aligned}
& \hat{y}_{1}=\tilde{\sigma}^{-1} \hat{\pi}_{2}+\hat{g}_{1} \\
& \hat{\pi}_{2}=\lambda\left(\hat{\pi}_{2}+\left[-\tilde{\kappa}_{y} \hat{y}_{1}+\tilde{\kappa}_{g} \hat{g}_{1}-\tilde{\kappa}_{\tau} \hat{\tau}_{1}\right]\right)
\end{aligned}
$$

Solving these simultaneously gives $\hat{y}_{1}$ and $\hat{\pi}_{2}$ :

$$
\begin{aligned}
& \hat{y}_{1}=\left(\frac{1+\tilde{\sigma}^{-1}\left(\frac{\lambda}{1-\lambda}\right) \tilde{\kappa}_{g}}{1+\tilde{\sigma}^{-1}\left(\frac{\lambda}{1-\lambda}\right) \tilde{\kappa}_{y}}\right) \hat{g}_{s}-\left(\frac{\tilde{\sigma}^{-1}\left(\frac{\lambda}{1-\lambda}\right) \tilde{\kappa}_{\tau}}{1+\tilde{\sigma}^{-1}\left(\frac{\lambda}{1-\lambda}\right) \tilde{\kappa}_{y}}\right) \hat{\tau}_{s} \\
& \hat{\kappa}_{2}=\left(\frac{\lambda}{1-\lambda}\right)\left[\tilde{\kappa}_{g}-\tilde{\kappa}_{y}\left(\frac{1+\tilde{\sigma}^{-1}\left(\frac{\lambda}{1-\lambda}\right) \tilde{\kappa}_{g}}{1+\tilde{\sigma}^{-1}\left(\frac{\lambda}{1-\lambda}\right) \tilde{\kappa}_{y}}\right)\right] \hat{g}_{s}+\left(\frac{\lambda}{1-\lambda}\right)\left[\tilde{\kappa}_{y}\left(\frac{\tilde{\sigma}^{-1}\left(\frac{\lambda}{1-\lambda}\right) \tilde{\kappa}_{\tau}}{1+\tilde{\sigma}^{-1}\left(\frac{\lambda}{1-\lambda}\right) \tilde{\kappa}_{y}}\right)-\tilde{\kappa}_{\tau}\right] \hat{\tau}_{s}
\end{aligned}
$$

Substituting $\hat{y}_{1}$ into period 1 Phillips curve (eqn 40) gives $\hat{\pi}_{1}$ :

$$
\hat{\pi}_{1}=\left(\frac{-\lambda}{1-\lambda}\right)\left[\tilde{\kappa}_{g}-\tilde{\kappa}_{y}\left(\frac{1+\tilde{\sigma}^{-1}\left(\frac{\lambda}{1-\lambda}\right) \tilde{\kappa}_{g}}{1+\tilde{\sigma}^{-1}\left(\frac{\lambda}{1-\lambda}\right) \tilde{\kappa}_{y}}\right)\right] \hat{g}_{s}+\left(\frac{-\lambda}{1-\lambda}\right)\left[\tilde{\kappa}_{y}\left(\frac{\tilde{\sigma}^{-1}\left(\frac{\lambda}{1-\lambda}\right) \tilde{\kappa}_{\tau}}{1+\tilde{\sigma}^{-1}\left(\frac{\lambda}{1-\lambda}\right) \tilde{\kappa}_{y}}\right)-\tilde{\kappa}_{\tau}\right] \hat{\tau}_{s}
$$

Further, from period 4 Phillips curve along with assumption (B3), and solution $\hat{y}_{3}=0$ we get:

$$
\begin{aligned}
0 & =\frac{\lambda}{1-\lambda}\left(\tilde{\kappa_{y}} \hat{y}_{4}\right)+\left[\lambda+\lambda(1-\lambda)+\lambda(1-\lambda)^{2}\right]\left(0+\tilde{\kappa_{y}}\left(\hat{y}_{4}-\hat{y}_{3}\right)\right) \\
\Longrightarrow \hat{y}_{4} & =\left[\frac{\lambda+\lambda(1-\lambda)+\lambda(1-\lambda)^{2}}{\frac{\lambda}{1-\lambda}+\lambda+\lambda(1-\lambda)+\lambda(1-\lambda)^{2}}\right] \hat{y}_{3} \\
& =0
\end{aligned}
$$

Similarly, we can solve for the transition dynamics of output in each period $t>2$ using the Phillips curve for that period along with the assumption (B3). For $t>2$, we get:

$$
\begin{aligned}
0 & =\frac{\lambda}{1-\lambda}\left(\tilde{\kappa_{y}} \hat{y}_{t}\right)+\lambda \Sigma_{j=0}^{t-2}(1-\lambda)^{j}\left(0+\tilde{\kappa_{y}}\left(\hat{y}_{t}-\hat{y}_{t-1}\right)\right) \\
0 & =\left[\frac{\lambda}{1-\lambda}+\lambda \Sigma_{j=0}^{\infty}(1-\lambda)^{j}\right] \tilde{\kappa_{y}} \hat{y}_{t}-\left[\lambda \Sigma_{j=0}^{t-2}(1-\lambda)^{j}\right] \tilde{\kappa_{y}} \hat{y}_{t-1} \\
\Longrightarrow \hat{y}_{t} & =\left[\frac{\lambda \Sigma_{j=0}^{t-2}(1-\lambda)^{j}}{\frac{\lambda}{1-\lambda}+\lambda \Sigma_{j=0}^{\infty}(1-\lambda)^{j}}\right] \hat{y}_{t-1} \\
& =0
\end{aligned}
$$




\section{Calibration parameters}

Table 7: Calibration parameters

\begin{tabular}{|c|c|c|}
\hline Common parameters & Description & Value \\
\hline$\beta$ & Discount factor & 0.9970 \\
\hline$\sigma$ & Risk aversion parameter & 1.032 \\
\hline$\eta$ & Frisch elasticity of labor supply & 1.7415 \\
\hline$\omega$ & Probability of price non-adjustment & 0.66 \\
\hline$\lambda$ & Probability of updating information & 0.15 \\
\hline$\theta$ & CES parameter & 13.6012 \\
\hline$\phi_{\pi}$ & Reaction to inflation & 100000 \\
\hline$\phi_{y}$ & Reaction to output gap & 0 \\
\hline$\psi$ & Steady state government spending to output ratio & 0.2 \\
\hline $\bar{\tau}$ & Steady state tax rate & 0.1 \\
\hline$\hat{r}_{s}^{e}$ & Generates $10 \%$ drop in output, $2 \%$ deflation & -0.0225 \\
\hline$\rho_{r n}$ & Makes ZLB bind for around 16 quarters & 0.88 \\
\hline Sticky-price parameter & & Value \\
\hline$\kappa$ & & 0.0841 \\
\hline$\kappa_{y}$ & & 0.0103 \\
\hline$\kappa_{g}$ & & 0.0044 \\
\hline$\kappa_{\tau}$ & & 0.0038 \\
\hline$\tilde{\sigma}$ & & 1.29 \\
\hline Sticky-information parameter & & Value \\
\hline$\tilde{\kappa}_{y}$ & & 0.1228 \\
\hline$\tilde{\kappa}_{g}$ & & 0.0523 \\
\hline$\tilde{\kappa}_{\tau}$ & & 0.0450 \\
\hline$\tilde{\sigma}$ & & 1.29 \\
\hline
\end{tabular}

Parameter details:

$\tilde{\sigma}=\frac{\sigma}{1-\psi}, \quad \psi=\frac{G}{Y}, \quad \kappa=\frac{(1-\omega)(1-\beta \omega)}{\omega}, \quad \kappa_{y}=\kappa(\tilde{\sigma}+\eta), \quad \kappa_{g}=\kappa \tilde{\sigma}, \quad \kappa_{\tau}=\frac{\kappa}{(1-\bar{\tau})}$,

$\tilde{\kappa}_{y}=\frac{\tilde{\sigma}+\eta}{1+\eta \theta}, \quad \tilde{\kappa}_{g}=\frac{\tilde{\sigma}}{1+\eta \theta}, \quad \tilde{\kappa}_{\tau}=\frac{1}{(1+\eta \theta)(1-\bar{\tau})}, \quad \hat{y}_{t}=\frac{Y_{t}-\bar{Y}}{\bar{Y}}, \quad \hat{g}_{t}=\frac{G_{t}-\bar{G}}{\bar{Y}}$,

$\hat{\tau}_{t}=\tau_{t}-\bar{\tau}, \quad \hat{\pi}_{t}=\hat{p}_{t}-\hat{p}_{t-1}, \quad \hat{i}_{t}=\frac{i_{t}-\bar{i}}{1+\bar{i}}, \quad \hat{r}_{t}^{e}=\hat{\xi}_{t}-\hat{\xi}_{t+1}=\frac{r_{t}^{e}-\bar{i}}{1+\bar{r}^{e}}$

\section{Coordinated Interest Rate Peg Experiment (CPEG-EX)}

The Coordinated-PEG experiment makes the following assumptions:

C1.(Shock): $\hat{r}_{t}^{e}=0 \quad \forall t, \quad \hat{i}_{1}=0$

C2.(Fiscal policy): $\left(\hat{g}_{1}, \hat{\tau}_{1}\right)=\left(\hat{g}_{s}, \hat{\tau}_{s}\right), \quad\left(\hat{g}_{2}, \hat{\tau}_{2}\right)=(0,0)$

C3.(Monetary policy): $\hat{\pi}_{t}=0$ for $t>1$

C4.(Perfect foresight): $\mathbb{E}_{t} \hat{y}_{t+1}=\hat{y}_{t+1}, \quad \mathbb{E}_{t} \hat{\pi}_{t+1}=\hat{\pi}_{t+1} \quad \forall t$

Sticky-price model

$$
\begin{aligned}
& \hat{y}_{t}=\mathbb{E}_{t} \hat{y}_{t+1}-\tilde{\sigma}^{-1}\left(\hat{i}_{t}-\mathbb{E}_{t} \hat{\pi}_{t+1}-\hat{r}_{t}^{e}\right)+\left(\hat{g}_{t}-\mathbb{E}_{t} \hat{g}_{t+1}\right) \\
& \hat{\pi}_{t}=\beta \mathbb{E}_{t} \hat{\pi}_{t+1}+\kappa_{y} \hat{y}_{t}-\kappa_{g} \hat{g}_{t}+\kappa_{\tau} \hat{\tau}_{t}
\end{aligned}
$$


Assumptions (C1)-(C4) imply that: For $\mathrm{t}=1$

$$
\begin{aligned}
& \hat{y}_{1}=\hat{y}_{2}+\hat{g}_{1} \\
& \hat{\pi}_{1}=\kappa_{y} \hat{y}_{1}-\kappa_{g} \hat{g}_{1}+\kappa_{\tau} \hat{\tau}_{1}
\end{aligned}
$$

For $\mathrm{t}=2$ :

$$
\begin{aligned}
& \hat{y}_{2}=\hat{y}_{3}-\tilde{\sigma}^{-1} \hat{i}_{2} \\
& \hat{\pi}_{2}=\kappa_{y} \hat{y}_{2}
\end{aligned}
$$

For $\mathrm{t}=3$ :

$$
\begin{aligned}
& \hat{y}_{3}=\hat{y}_{4}-\tilde{\sigma}^{-1} \hat{i}_{3} \\
& \hat{\pi}_{3}=\kappa_{y} \hat{y}_{3}
\end{aligned}
$$

For $\mathrm{t}>2$ :

$$
\begin{aligned}
& \hat{y}_{t}=\hat{y}_{t+1}-\tilde{\sigma}^{-1} \hat{i}_{t} \\
& \hat{\pi}_{t}=\kappa_{y} \hat{y}_{t}
\end{aligned}
$$

Applying (C3) to period 2 Phillips curve (eqn 54) allows us to solve for $\hat{y}_{2}$ as follows:

$$
\begin{aligned}
\hat{\pi}_{2} & =\kappa_{y} \hat{y}_{2} \\
0 & =\kappa_{y} \hat{y}_{2} \\
\Longrightarrow \hat{y}_{2} & =0
\end{aligned}
$$

Substituting $\hat{y}_{2}$ into period 1 IS curve (eqn 53) gives:

$$
\hat{y}_{1}=\hat{g}_{1}
$$

Substituting $\hat{y}_{1}$ in period 1 Phillips curve (eqn 53) gives:

$$
\hat{\pi}_{1}=\left(\kappa_{y}-\kappa_{g}\right) \hat{g}_{1}+\kappa_{\tau} \hat{\tau}_{1}
$$

Output for every period $t>2$ can be solved from that period's Phillips curve using assumption (C3). We get that for $\mathrm{t}>2, \hat{y}_{t}=\hat{\pi}_{t}=0$.

\section{Sticky-information model}

$$
\begin{aligned}
\hat{y}_{t} & =\mathbb{E}_{t} \hat{y}_{t+1}-\tilde{\sigma}^{-1}\left(\hat{i}_{t}-\mathbb{E}_{t} \hat{\pi}_{t+1}-\hat{r}_{t}^{e}\right)+\left(\hat{g}_{t}-\mathbb{E}_{t} \hat{g}_{t+1}\right) \\
\hat{\pi}_{t} & =\frac{\lambda}{1-\lambda}\left(\tilde{\kappa}_{y} \hat{y}_{t}-\tilde{\kappa}_{g} \hat{g}_{t}+\tilde{\kappa}_{\tau} \hat{\tau}_{t}\right) \\
& +\lambda \Sigma_{j=0}^{\infty}(1-\lambda)^{j} \mathbb{E}_{t-1-j}\left(\hat{\pi}_{t}+\left[\tilde{\kappa}_{y}\left(\hat{y}_{t}-\hat{y}_{t-1}\right)-\tilde{\kappa}_{g}\left(\hat{g}_{t}-\hat{g}_{t-1}\right)+\tilde{\kappa}_{\tau}\left(\hat{\tau}_{t}-\hat{\tau}_{t-1}\right)\right]\right)
\end{aligned}
$$

Assumptions (C1)-(C4) imply that: For $\mathrm{t}=1$

$$
\begin{aligned}
& \hat{y}_{1}=\hat{y}_{2}+\hat{g}_{1} \\
& \hat{\pi}_{1}=\frac{\lambda}{1-\lambda}\left(\tilde{\kappa}_{y} \hat{y}_{1}-\tilde{\kappa}_{g} \hat{g}_{1}+\tilde{\kappa}_{\tau} \hat{\tau}_{1}\right)
\end{aligned}
$$

For $\mathrm{t}=2$ :

$$
\begin{aligned}
& \hat{y}_{2}=\hat{y}_{3}-\tilde{\sigma}^{-1} \hat{i}_{2} \\
& \hat{\pi}_{2}=\frac{\lambda}{1-\lambda} \tilde{\kappa}_{y} \hat{y}_{2}+\lambda\left(\hat{\pi}_{2}+\left[\tilde{\kappa}_{y}\left(\hat{y}_{2}-\hat{y}_{1}\right)+\tilde{\kappa}_{g} \hat{g}_{1}-\tilde{\kappa}_{\tau} \hat{\tau}_{1}\right]\right)
\end{aligned}
$$


For $\mathrm{t}=3$ :

$$
\begin{aligned}
& \hat{y}_{3}=\hat{y}_{4}-\tilde{\sigma}^{-1} \hat{i}_{3} \\
& \hat{\pi}_{3}=\frac{\lambda}{1-\lambda} \tilde{\kappa}_{y} \hat{y}_{3}+[\lambda+\lambda(1-\lambda)]\left(\hat{\pi}_{2}+\tilde{\kappa}_{y}\left(\hat{y}_{3}-\hat{y}_{2}\right)\right)
\end{aligned}
$$

For $\mathrm{t}=4$ :

$$
\begin{aligned}
& \hat{y}_{4}=\hat{y}_{5}-\tilde{\sigma}^{-1} \hat{i}_{4} \\
& \hat{\pi}_{4}=\frac{\lambda}{1-\lambda} \tilde{\kappa}_{y} \hat{y}_{4}+\left[\lambda+\lambda(1-\lambda)+\lambda(1-\lambda)^{2}\right]\left(\hat{\pi}_{4}+\tilde{\kappa}_{y}\left(\hat{y}_{4}-\hat{y}_{3}\right)\right)
\end{aligned}
$$

For $\mathrm{t}>2$ :

$$
\begin{aligned}
\hat{y}_{t} & =\hat{y}_{t+1}-\tilde{\sigma}^{-1} \hat{i}_{t} \\
\hat{\pi}_{t} & =\frac{\lambda}{1-\lambda} \tilde{\kappa}_{y} \hat{y}_{t} \\
& +\lambda \Sigma_{j=0}^{t-2}(1-\lambda)^{j}\left(\hat{\pi}_{t}+\tilde{\kappa}_{y}\left(\hat{y}_{t}-\hat{y}_{t-1}\right)\right)
\end{aligned}
$$

Period 2 Phillips curve (eqn 62) along with assumption (C3) can be used to solve for $\hat{y}_{2}$ as follows:

$$
\begin{aligned}
\hat{\kappa}_{2} & =\frac{\lambda}{1-\lambda} \tilde{\kappa}_{y} \hat{y}_{2}+\lambda\left(\hat{\kappa}_{2}+\left[\tilde{\kappa}_{y}\left(\hat{y}_{2}-\hat{y}_{1}\right)+\tilde{\kappa}_{g} \hat{g}_{1}-\tilde{\kappa}_{\tau} \hat{\tau}_{1}\right]\right) \\
0 & =\frac{\lambda}{1-\lambda} \tilde{\kappa}_{y} \hat{y}_{2}+\lambda\left(0+\left[\tilde{\kappa}_{y}\left(\hat{y}_{2}-\hat{y}_{1}\right)+\tilde{\kappa}_{g} \hat{g}_{1}-\tilde{\kappa}_{\tau} \hat{\tau}_{1}\right]\right) \\
\hat{y}_{2} & =\left(\frac{1-\lambda}{2-\lambda}\right)\left[\hat{y}_{1}-\frac{\tilde{\kappa}_{g}}{\tilde{\kappa}_{y}} \hat{g}_{1}+\frac{\tilde{\kappa}_{\tau}}{\tilde{\kappa}_{y}} \hat{\tau}_{1}\right]
\end{aligned}
$$

Substituting $\hat{y}_{2}$ into period 1 IS curve (eqn 61) gives $\hat{y}_{1}$ :

$$
\hat{y}_{1}=\left[\frac{(2-\lambda) \tilde{\kappa}_{y}-(1-\lambda) \tilde{\kappa}_{g}}{\tilde{\kappa}_{y}}\right] \hat{g}_{1}+\left[\frac{(1-\lambda) \tilde{\kappa}_{\tau}}{\tilde{\kappa}_{y}}\right] \hat{\tau}_{1}
$$

Proposition 3 (Government spending multipliers). In the CPEG-EX, the government spending multiplier in the sticky-information model is bounded below by 1 while it is equal to 1 in the sticky-price model.

$$
\begin{aligned}
& \left(\frac{d \hat{y}_{1}}{d \hat{g}_{1}}\right)^{S I}=\frac{(2-\lambda) \tilde{\kappa}_{y}-(1-\lambda) \tilde{\kappa}_{g}}{\tilde{\kappa}_{y}} \geq 1 \\
& \left(\frac{d \hat{y}_{1}}{d \hat{g}_{1}}\right)^{S P}=1
\end{aligned}
$$

Proposition 4 (Paradox of toil). In the CPEG-EX, tax cuts are contractionary in the sticky-information model but have no effect in the sticky-price model.

$$
\begin{aligned}
& \left(\frac{d \hat{y}_{1}}{d \hat{\tau}_{1}}\right)^{S I}=\frac{(1-\lambda) \tilde{\kappa}_{\tau}}{\tilde{\kappa}_{y}} \geq 0 \\
& \left(\frac{d \hat{y}_{1}}{d \hat{\tau}_{1}}\right)^{S P}=0
\end{aligned}
$$


Numerical results: ZLB-EX vs CPEG-EX

Table 8: Fiscal multipliers under sticky-prices

\begin{tabular}{lll}
\hline & Government spending & Tax cut \\
\hline ZLB-EX & 1.63 & -0.4 \\
CPEG-EX & 1.04 & -0.03 \\
\hline
\end{tabular}

Table 9: Fiscal multipliers under sticky-information

\begin{tabular}{lll}
\hline & Government spending & Tax cut \\
\hline ZLB-EX & 4.77 & -2.41 \\
CPEG-EX & 5.14 & -2.64 \\
\hline
\end{tabular}

